\title{
L'outil idéal. Analyse du standard Levallois des sites moustériens d'Hermies (Nord de la France)
}

The perfect tool. Analysis of the Levallois pattern in the Mousterian settlements of Hermies (North of France)

Luc Vallin, Bertrand Masson, Jean-Paul Caspar et Éric Depiereux

\section{OpenEdition}

\section{Journals}

Édition électronique

URL : http://journals.openedition.org/paleo/347

DOI : 10.4000/paleo.347

ISSN : 2101-0420

\section{Éditeur}

SAMRA

\section{Édition imprimée}

Date de publication : 1 décembre 2006

Pagination : 237-272

ISSN : 1145-3370

\section{Référence électronique}

Luc Vallin, Bertrand Masson, Jean-Paul Caspar et Éric Depiereux, «L'outil idéal. Analyse du standard Levallois des sites moustériens d'Hermies (Nord de la France) », PALEO [En ligne], 18 | 2006, mis en ligne le 23 avril 2009, consulté le 07 juillet 2020. URL : http://journals.openedition.org/paleo/347 ; DOl : https://doi.org/10.4000/paleo.347

\section{(c) (i) $\Theta$}

PALEO est mis à disposition selon les termes de la licence Creative Commons Attribution - Pas d'Utilisation Commerciale - Pas de Modification 4.0 International 


\title{
L'OUTIL IDÉAL. \\ ANALYSE DU STANDARD LEVALLOIS DES SITES MOUSTÉRIENS D'HERMIES (Nord de la France)
}

\author{
Luc VALLIN ${ }^{(1)}$, Bertrand MASSON ${ }^{(1)}$, Jean-Paul CASPAR ${ }^{(2)}$ et Éric DEPIEREUX ${ }^{(3)}$
}

\begin{abstract}
Résumé : Des fouilles récentes sur deux gisements moustériens de plein air du nord de la France ont mis au jour plusieurs niveaux d'occupation bien conservés où des activités de taille ont été conduites au sein de postes de débitage clairement délimités. Le débitage du silex, d'origine locale, était totalement orienté vers la production d'éclats préférentiels selon un schéma Levallois linéal. En confrontant les données fournies par différentes approches (analyse technologique, remontages, analyse spatiale, tracéologie) nous avons tenté d'identifier les produits finis répondant aux exigences des utilisateurs. Une analyse statistique portant sur la quasi-totalité des produits finis, soit 127 éclats préférentiels, a montré que les critères de sélection des pièces produites à des fins d'usage de boucherie se limitaient essentiellement au choix des éclats préférentiels présentant un profil longitudinal strictement rectiligne. La production, bien que fortement normalisée, admettait une certaine variabilité dimensionnelle ; au sein de cette gamme, les utilisateurs ont, dans un des niveaux, sélectionné deux formats assez lâches correspondant à deux types d'utilisation distincts. On pose également le problème de l'économie du débitage et des rapports entre la production effectuée sur place et les produits importés.
\end{abstract}

Mots-clés : amas de débitage, analyse fonctionnelle, analyse multivariée, analyse univariée, analyse spatiale, Levallois, Moustérien, nord de la France, outil, technologie lithique.

Key-words : knapping cluster, functional analysis, multivariate analysis, univariate analysis, spatial analysis, Levallois, Mousterian, north of France, tool, lithic technology.

\author{
Abridged english version

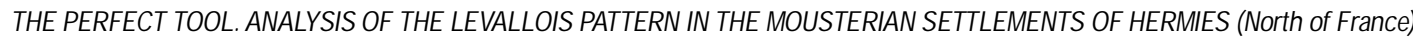

Our aim is to examine the degree of standarization of the endproducts produced by a Levallois lineal debitage and to characterize the constraint experienced by the Middle Palaeolithic knappers throughout the "chaîne opératoire ", from the raw material procurement to the use of tools.

Two mousterian open air settlements ("Champ Bruquette " and "Tio Marché ") have been recently excavated (1993-2003) in the loess of northem France at Hermies (Pas-de-Calais) ; several archaeological levels were recognized : they belong to the Last Glacial, from the OIS $5 \mathrm{~d}$ to OIS 3 . On each site, one well presened occupation floor showed knapping areas ; technological analysis, reffiting, spatial analysis and traceology have proved that only some unretouched preferential flakes were used. All preferential flakes ( 28 from Champ Bruquette, 99 from Tio Marché) were captured in a database in order to establish the use criteria.

A first selection was made by Mousterian knappers who chose the local raw material from a bed of gravels, in spite of its inferior quality, and selected the favourable shapes among the biggest flint nodules (table 1). The size of the selected blocks varies from 15 to 30 $\mathrm{cm}$ (fig. 1). These blocks were knapped on and near the outcrop, without solution of continuity.

The knapping scatters vary in size : one to about 20 reduction sequences were performed in these places; most of the debitages belong to the Levallois lineal method (table 2, fig. 5, 6, 7, 8). The Levallois surface was often roughly shaped and the frequent dissimmetry of the sides (fig. 2) produced some plunging flakes and "eclats debordants " (fig. 3). When possible, the Levallois core was reprepared to obtain a second, third or even fourth preferential flake (fig. 4); these next flakes are paradoxically larger than the first one, because they are more invasive (fig. 9, 10, 11, 12). Few Levallois cores were trimmed by bipolar debitage (fig. 5). A minority of nonLevallois debitages was recognized : some of them were performed on recycled fragments of broken blocks or cores, the other

(1) Ministère de la Culture et de la Communication, Service Régional de l'Archéologie du Nord-Pas-de-Calais, Ferme Saint-Sauveur, avenue du Bois, 59650 Villeneuve d'Ascq - luc.vallin@culture.gouv.fr

(2) Facultés Universitaires Notre-Dame de la Paix, rue de Bruxelles, 61, 5000 Namur (Belgique).

(3) Facultés Universitaires Notre-Dame de la Paix, Laboratoire de Biostatistiques et Bioinformatique, rue de Bruxelles,

61, 5000 Namur (Belgique) - eric.depiereux@fundp.ac.be 
are roughly done, maybe by young people.

The endproducts show a relative diversity of features ; some of them depend from the particular preparation of the core (fig. 13, 14). The classification of the preferential flakes according to the angle of edge (table 3), the shape (table 4), the size (table 5, fig. 17) and the length of cutting edge (fig. 18) displays a slight variability about an ovary pattern (fig. 19).

Two methods have been employed for identifying the used flakes. The traceology (Dr Jean-Paul Capar) has distinguished 9 flakes which show an edge with removals due to the percussion on soft mineral or hard organic material (fig. 21) ; 12 other flakes wear micropolishes : two have probably been subjected to postdepositional processes, but ten have been certainly used for butchery activities (fig. 20). The good preservation of the main archaeological floors and the conservation of in situ knapping areas allowed to classify the endproducts in three main groups and five subgroups (fig. 22, table 6) :

- group $A$ : the preferential flake remained in the debitage area where he was knapped;

- group B : the preferential flake was left outside of the debitage area : in an other debitage area (B1) or off any debitage area (B2) ;

- group $C$ : the preferential flake has been found off the debitage area where it was produced, the location of which is unknown; like group $B$, it may stay in an other debitage area (C1) or off any debitage area (C2) ;

- group D has not been considered ; it includes the lacking preferential flakes, the scar of which remains on the cores.

The groups A and B are interpreted as the domestic production, the group C includes the "imported " flakes. The probability of use concerns groups B, C (and D), that is confirmed by the use wear analysis : most of the used flakes belong to subgroup C2.

The preferential flakes have been submitted to univariate and multivariate analysis (Prof. Eric Depiereux) that have displayed the importance of the profile of the flakes (table 7): almost all the used flakes showed a rectilinear profile. The presence of small removals on the edges near the butt of used flakes is a good criterion of diagnosis (fig. 24) ; the location of these retouchs on the opposite side to the used edge suggests that flakes were hand-held, although some microscopic traces on several used flakes show possible hafting. Few differences appear beetween the two sites (table 8), except the lack of flakes used on soft mineral or hard organic material at Champ Bruquette and the slightly bader quality of raw material at Champ Bruquette. The multivariate analysis has allowed to suggest a possible use for some flakes from both sites which show no use mark (table 9) : the best probability of use concerns flakes belonging to subgroups $\mathrm{C} 1$ and $\mathrm{C} 2$.

We conclude that Mousterian at Hermies restricted the use to only preferential flakes, among them they selected the most regular items, showing a rectilinear profile. The relative variability in size allowed to select, at least at Tio Marché, two distinct grades for two specific uses (fig. 23) but it seems that this grading was not really thought ahead. The question is so the relationship beetween production and use, because most of the knapped preferential flakes were considered as unsuited to use and left on the knapping spot. Conversely, the largest and the better preferential flakes, on both sites, were introduced from other workshops, that suggests the possibility of exchange network.

Finally, we emphasize the specialization of some recent Middle Paleolithic sites in one particular schema operatoire, either Levallois or discoid method. The chronological signifiance of this preference is discussed, because the Levallois method predominates all along the stratigraphy at Hermies.

\section{1 - INTRODUCTION}

Les deux gisements moustériens d'Hermies " le Champ Bruquettte " et Hermies "le Tio Marché " se situent dans une petite vallée sèche du nord de la France (département du Pas-de-Calais), à 900 mètres l'un de l'autre, sur le même versant vers $85 \mathrm{~m}$ d'altitude. Ils ont été découverts au début du XXème siècle, à l'occasion du creusement du Canal du Nord qui les a amputés d'une partie de leur superficie (Salomon 1913). Des fouilles y ont été conduites entre 1993 et 2003 , dans le cadre d'une recherche programmée. Deux traits essentiels caractérisent ces gisements dont les niveaux s'étendent au moins du début du Weichsélien ancien (stade isotopique $5 \mathrm{~d}$ ?) au Pléniglaciaire moyen (stade 3 ) inclus. La première particularité réside dans l'excellent état de préservation des niveaux principaux, tant du point de vue de l'état physique des artefacts en silex, particulièrement propice à une étude tracéologique, que de la conservation des sols archéologiques, propice à une analyse spatiale fine (Masson et Vallin 1993, 1996 ; Vallin et Masson 2000, 2004 ; Vallin et al. 2001). La seconde caractéristique est la rigidité du cadre technologique des productions lithiques, marquée par la permanence d'un débitage Levallois linéal prédominant, voire quasi-exclusif (Vallin et Masson 1996). Ces conditions offrent l'opportunité parfaite d'une réflexion sur le schéma mental sous-tendu par le débitage Levallois et sur sa contrainte, à savoir si ce cadre est ouvert ou fermé (Van Peer 1992 ; Dibble et Bar-Yosef 1995) ; en d'autres termes, quel est le degré de liberté offert au tailleur, quel potentiel d'évolution est révélé par la confrontation de niveaux diachrones ? La définition du schéma Levallois renvoie au concept de prédétermination (Tixier 1959 ; Bordes 1961 ; Heinzelin de Braucourt 1962 ; Boëda 1994) : on peut rechercher quel est le degré d'abstraction qu'il représente, en mesurant jusqu'où va l'adaptation des méthodes aux objectifs (Sandgathe 2005).

Parallèlement à la lecture technologique des schémas opératoires (fournie par les nombreux remontages), nous avons utilisé une base de données intégrant les éclats Levallois préférentiels provenant des deux sites, à l'exclusion des fragments non raccordés et des éclats dont la détermination pouvait prêter à discussion. Cela représentait, à la date de rédaction de l'article ${ }^{4}, 28$ individus pour le Champ Bruquette (5 provenant du niveau $b$, sous-jacent au complexe de sols humifères du Début Glaciaire weichsélien, 22 provenant du

(4) Cet article est issu d'une communication présentée dans le cadre de la 5e session du XIVème Congrès de I'U.I.S.P.P. tenu à Liège en septembre 2001. 
niveau a, attribué au stade isotopique 4 , et 1 provenant d'un loess pléniglaciaire du Weichsélien) et 99 individus pour le Tio Marché (8 provenant du cailloutis de base et 91 provenant des horizons attribués au stade isotopique 3 , qui représentent ainsi la plus grosse partie de la population soumise à l'étude). Un certain nombre d'éclats préférentiels entiers, provenant surtout des cailloutis des deux sites, n'ont pas été pris en compte parce que leur bord était trop ébréché, rendant leur délinéation et leur angulation inidentifiables. La structure de la base de données est exposée en annexe. Nous nous proposons de mesurer, à chaque stade de la chaîne opératoire, le degré de standardisation sous-tendant les choix des préhistoriques d'Hermies, dont on peut supposer l'appartenance au groupe des derniers Néandertaliens.

\section{2 - STANDARDISATION DE LA PRODUCTION}

\section{1 - Standardisation de la matière première}

Le premier choix du tailleur intervient lors de la phase d'acquisition de la matière première. Plusieurs ressources étaient offertes aux tailleurs d'Hermies dans le voisinage immédiat des deux sites:

- en position primaire, le silex du Turonien supérieur et le silex coniacien (Sénonien) affleurent actuellement, sous forme de lits de rognons subhorizontaux, dans le versant de la vallée d'Hermies exposé au sud ;

- en position secondaire, le silex est abondant dans le cailloutis, épais de plusieurs décimètres, qui tapisse le versant opposé, en pente douce, sur lequel se sont établis les préhistoriques ; ce cailloutis devait affleurer au débouché des vallons affluents, ce qui a probablement guidé le choix des installations (Commont 1916). II est essentiellement constitué d'éléments provenant de l'altération sur place du Sénonien. II présente des caractères identiques sur les deux sites.

Le silex en provenance directe de la craie a été très peu ou pas exploité, en dehors d'un très petit nombre de produits qui n'ont probablement pas été débités sur place. Les Moustériens d'Hermies, quels que soient le site et le niveau considérés, ont porté leur choix sur le silex du cailloutis local. L'abondance et la disponibilité compensaient certainement la qualité souvent médiocre de la matière première, affectée de fissures dues au gel et renfermant fréquemment

\begin{tabular}{|l|c|c|}
\hline & $\begin{array}{l}\text { Rognons bruts du cailloutis } \\
\text { (Tio Marché, } \mathrm{n}=100)\end{array}$ & $\begin{array}{l}\text { Rognons exploités } \\
\text { par les Moustériens } \\
\text { (Champ Bruquette, } \mathrm{n}=25)\end{array}$ \\
\hline Informe ou biscornu & $13,3 \%$ & $24,0 \%$ \\
\hline Discoïde & $26,7 \%$ & $8,0 \%$ \\
\hline Globuleux & $23,3 \%$ & $32,0 \%$ \\
\hline Cylindrique ou fusiforme & $26,3 \%$ & $32,0 \%$ \\
\hline Autre & $10,4 \%$ & $4,0 \%$ \\
\hline
\end{tabular}

Tableau 1 - Classement morphologique des plus gros rognons bruts du cailloutis de base d'Hermies-Le Tio Marché et des rognons exploités du niveau a d'Hermies-Le Champ Bruquette.

Table 1 - Morphological classification of the largest rough blocks from the gravels layer at Hermies-Le Tio Marché and the knapped blocks from the level a at Hermies-Le Champ Bruquette.

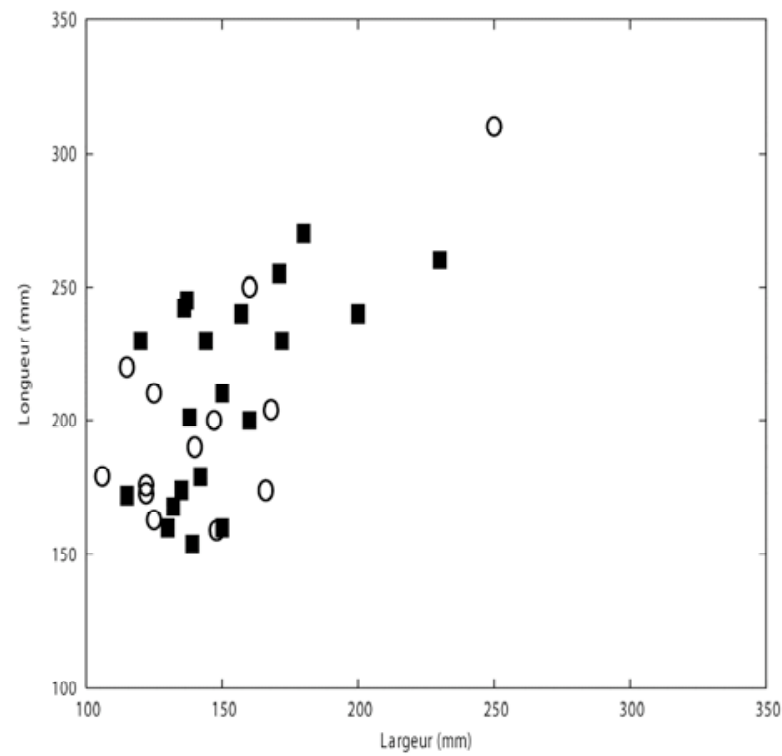

Figure 1 - Classement dimensionnel des rognons exploités du niveau a d'Hermies-le Champ Bruquette (symbolisés par des carrés noirs, $n=22)$ et du niveau pléniglaciaire moyen d'Hermies-le Tio Marché (symboles blancs, $n=13$ ).

Figure 1 - Size sorting of knapped blocks from Hermies-le Champ Bruquette (symbolised by black squares, $n=22$ ) and from the Middle Pleniglacial level from Hermies-le Tio Marché (symbolised by black squares, $n=13$ ). 
des géodes, des cristallisations et des zones mal silicifiées, plurimillimétriques, centimétriques ou pluricentimétriques. En dehors de ces accidents, le grain du silex est plutôt fin. La taille des éléments du cailloutis est assez variable, ainsi que leur forme (tabl. 1). Les graviers sont majoritaires, les cailloux supérieurs à $15 \mathrm{~cm}$ sont rares, leur densité varie selon les secteurs mais ils représentent toujours moins de 7 $\%$ des éléments supérieurs à $20 \mathrm{~mm}$ en surface du cailloutis de base (tant au Tio Marché qu'au Champ Bruquette). L'examen des blocs remontés, qui présentent une certaine homogénéité morphologique et dimensionnelle, montre une sélection des tailleurs et permet de définir un premier niveau d'exigence. La norme est celle d'un bloc subcylindrique, fusiforme ou globuleux, légèrement allongé, mesurant entre 15 et $30 \mathrm{~cm}$ dans sa plus grande dimension (tabl. 1, fig. 1) et d'un poids oscillant autour de 2,3 $\mathrm{kg}$. Les rognons de morphologie discoïde ont été plus rarement sélectionnés, sans doute en raison de leur épaisseur moindre, les rendant inaptes à un débitage Levallois linéal pluriséquentiel. Les blocs informes ou biscornus, une fois débarrassés de leurs protubérances, prenaient une forme globuleuse.

Il existe aussi, au sein du cailloutis qui est localement stratifié, des variations qualitatives qui étaient connues des tailleurs puisqu'une certaine sélection a été opérée, sur le site du Tio Marché, en faveur de gros rognons globuleux $(>3 \mathrm{~kg})$ de silex noir non fissuré à structure vitreuse et cortex épais, dont le gîte se situe dans le fond du vallon bordant le site : ils ont été réservés aux débitages les plus soignés ; ces blocs se distinguent par des dimensions nettement plus élevées, de l'ordre de $30 \mathrm{~cm}$ (fig. 1).

\section{2 - Standardisation des méthodes}

Les activités de débitage du silex conduites sur les deux sites se sont déroulées au sein de postes de débi- tage de dimension et de densité variables totalisant, chacun, de une à près de 20 séquences de réduction. Les amas en résultant présentent, pour la plupart, une consenation excellente. La plus grande partie des produits issus du débitage est demeurée concentrée sur le lieu de taille et les remontages sont suffisamment complets pour autoriser une perception fine des objectifs et des méthodes des tailleurs, complétant ou corrigeant la lecture diacritique des nucléus (Geneste 1985) ; dans certains secteurs des deux sites, toutefois, les postes de débitage ont été dispersés à divers degrés (cf. infra). Le débitage Levallois linéal prédomine largement dans les niveaux principaux, ce qui permet de ne pas tenir compte d'un éventuel diachronisme entre les amas d'un même niveau (tabl. 2). La principale objection au bienfondé de l'analyse technologique que nous proposons pourrait provenir d'une éventuelle structuration de l'espace en fonction des méthodes de débitage ; il serait toutefois peu vraisemblable que d'autres chaînes opératoires soient entièrement restreintes aux seules zones non fouillées sur les deux sites, particulièrenent au Tio Marché où la surface fouillée atteint $503 \mathrm{~m}^{2}$ et la surface explorée près de $700 \mathrm{~m}^{2}$.

Tous les intermédiaires existent entre le bloc brut et le nucléus débité, ce qui permet d'aborder certaines questions techniques, comme le rapport entre la forme des blocs et les méthodes de débitage ou bien les raisons de l'abandon d'un bloc, aux différents stades de la chaîne opératoire.

La proportion de nucléus ébauchés est relativement faible, on peut l'estimer, au Tio Marché (niveaux supérieurs), à un peu plus de $10 \%$ des rognons débités ; il s'agit le plus souvent, pour autant qu'on puisse en juger, de préparation en vue d'un débitage Levallois. Les tailleurs ont procédé par enlèvements alternants centripètes sur une partie plus

\begin{tabular}{|l|c|c|c|c|c|c|c|}
\hline $\begin{array}{l}\text { Niveau } \\
\text { d'occupation, par } \\
\text { ordre chronologique }\end{array}$ & $\begin{array}{l}\text { Nombre } \\
\text { d'éclats } \\
\text { préférentiels }\end{array}$ & $\begin{array}{l}\text { Total nucléus } \\
\text { déterminés } \\
\text { (NR) }\end{array}$ & $\begin{array}{l}\text { Nucléus } \\
\text { Levallois } \\
\text { linéal }\end{array}$ & $\begin{array}{l}\text { Nucléus } \\
\text { Levallois } \\
\text { récurrent }\end{array}$ & $\begin{array}{l}\text { Nucléus } \\
\text { discoïde }\end{array}$ & $\begin{array}{l}\text { Nucléus } \\
\text { laminaire } \\
\text { volumétrique }\end{array}$ & $\begin{array}{l}\text { Biface } \\
(\mathrm{n})\end{array}$ \\
\hline $\begin{array}{l}\text { HTM, complexe de } \\
\text { Saint-Acheul }\end{array}$ & 138 & 118 & 108 & 3 & 1 & 0 & 0 \\
\hline $\begin{array}{l}\text { HCB, loess du } \\
\text { Pléniglaciaire inf. } \\
\text { (niveau a) }\end{array}$ & 22 & 39 & 32 & 1 & 0 & 0 & 0 \\
\hline $\begin{array}{l}\text { HTM, sol du Début } \\
\text { Glaciaire }\end{array}$ & 1 & 1 & 0 & 0 & 0 & 1 & 0 \\
\hline $\begin{array}{l}\text { HCB, surface du } \\
\text { cailloutis (niveau b) }\end{array}$ & 5 & 41 & 13 & 4 & 0 & 0 & 0 \\
\hline HCB, cailloutis & 4 & 17 & 11 & 4 & 0 & 0 & 0 \\
\hline HTM, cailloutis & 12 & 60 & 59 & 0 & 1 & 0 & 2 \\
\hline
\end{tabular}

Tableau 2 - Fréquence de la production Levallois, par niveau. HTM = Hermies-Le Tio Marché, HCB = Hermies-Le Champ Bruquette. NB : les ébauches et les blocs testés ne sont pas comptabilisés dans les nucléus.

Table 2 - Levallois production frequency by level. HTM = Hermies-Le Tio Marché, HCB = Hermies-Le Champ Bruquette. NB : tested blocks have not been taken into account. 

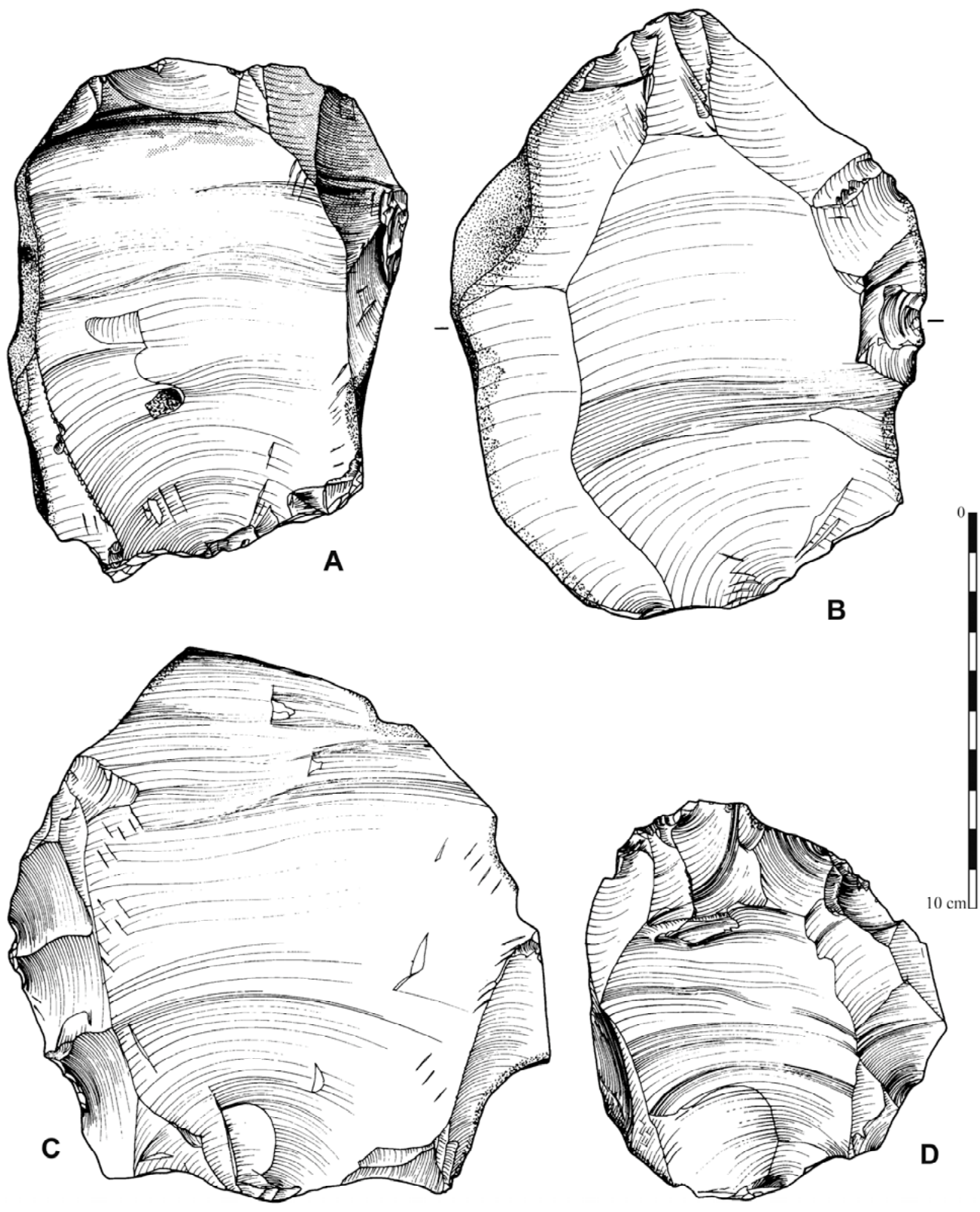

Figure 2 - Nucléus Levallois d'Hermies-Le Champ Bruquette : A-B99/343, B-A98/25, C-A98/1, D-B100/8.

Figure 2 - Levallois cores from Hermies-Le Champ Bruquette : A-B99/343, B-A98/25, C-A98/1, D-B100/8. 


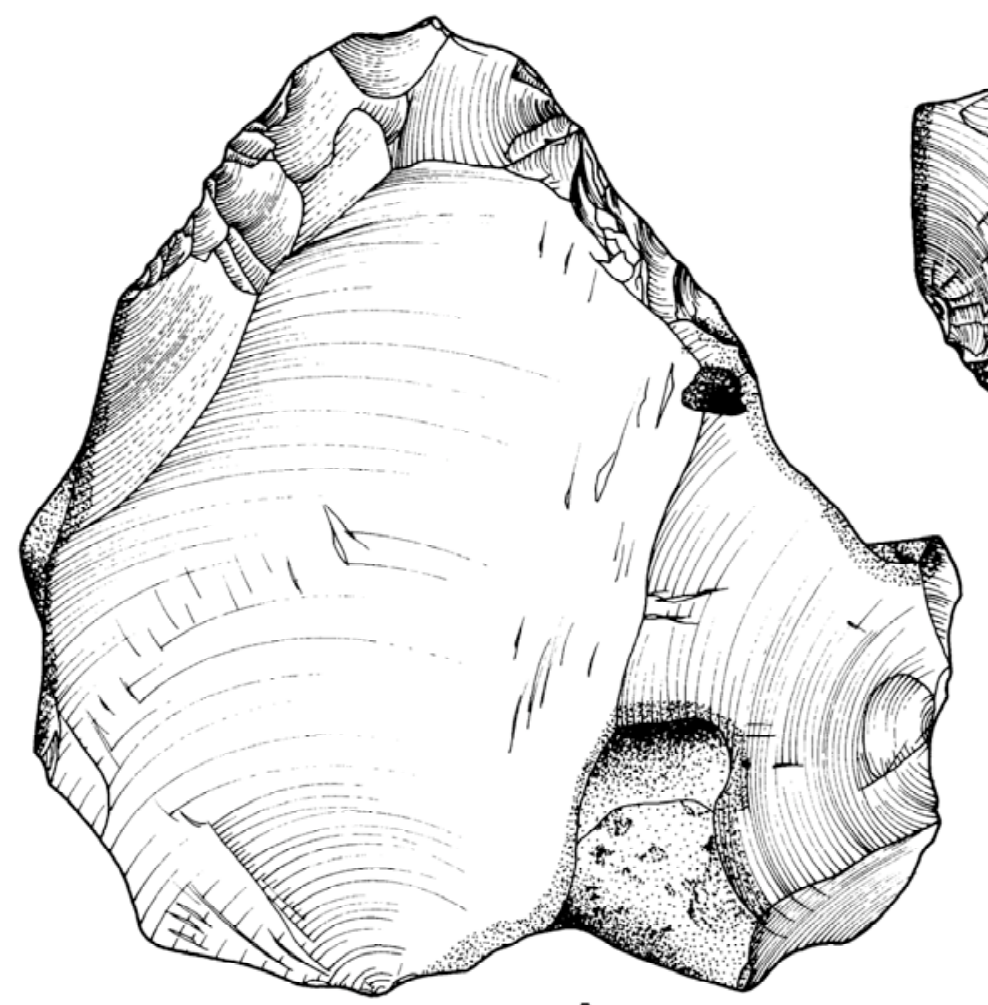

A

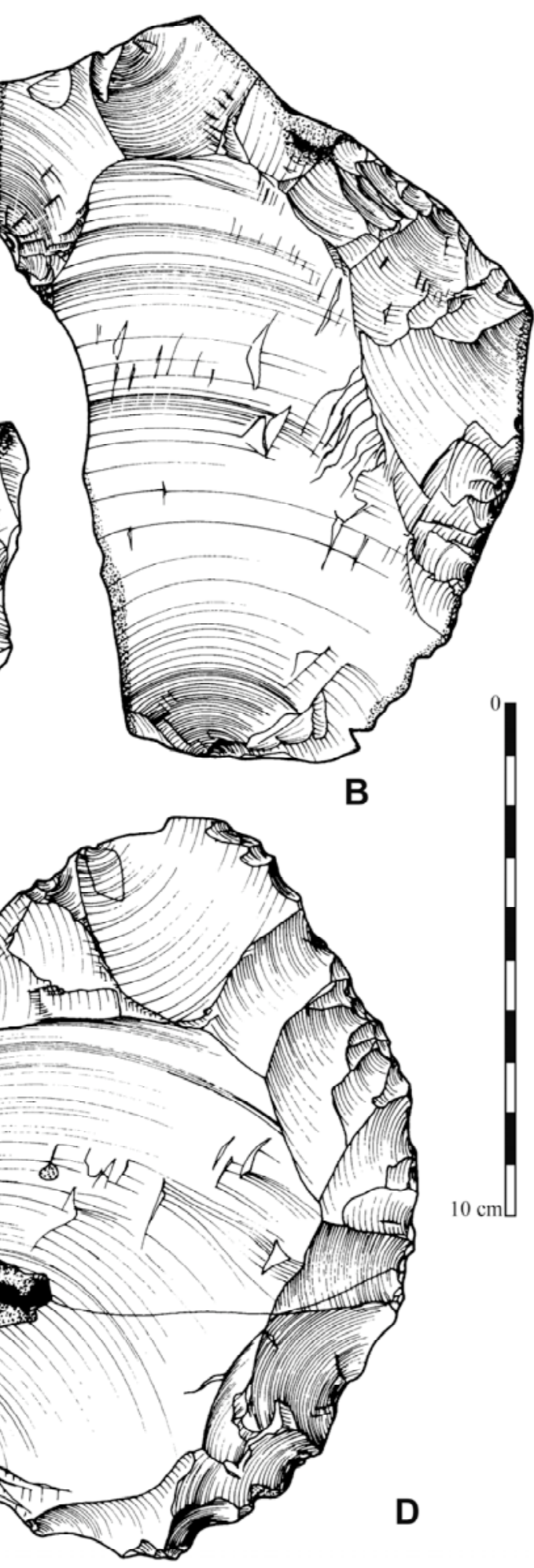

Figure 3 - Nucléus Levallois d'Hermies-Le Champ Bruquette : A-A98/169, B-B99/100, C-B100/22, D-B98/12.

Figure 3 - Levallois cores from Hermies-Le Champ Bruquette : A-A98/169, B-B99/100, C-B100/22, D-B98/12. 

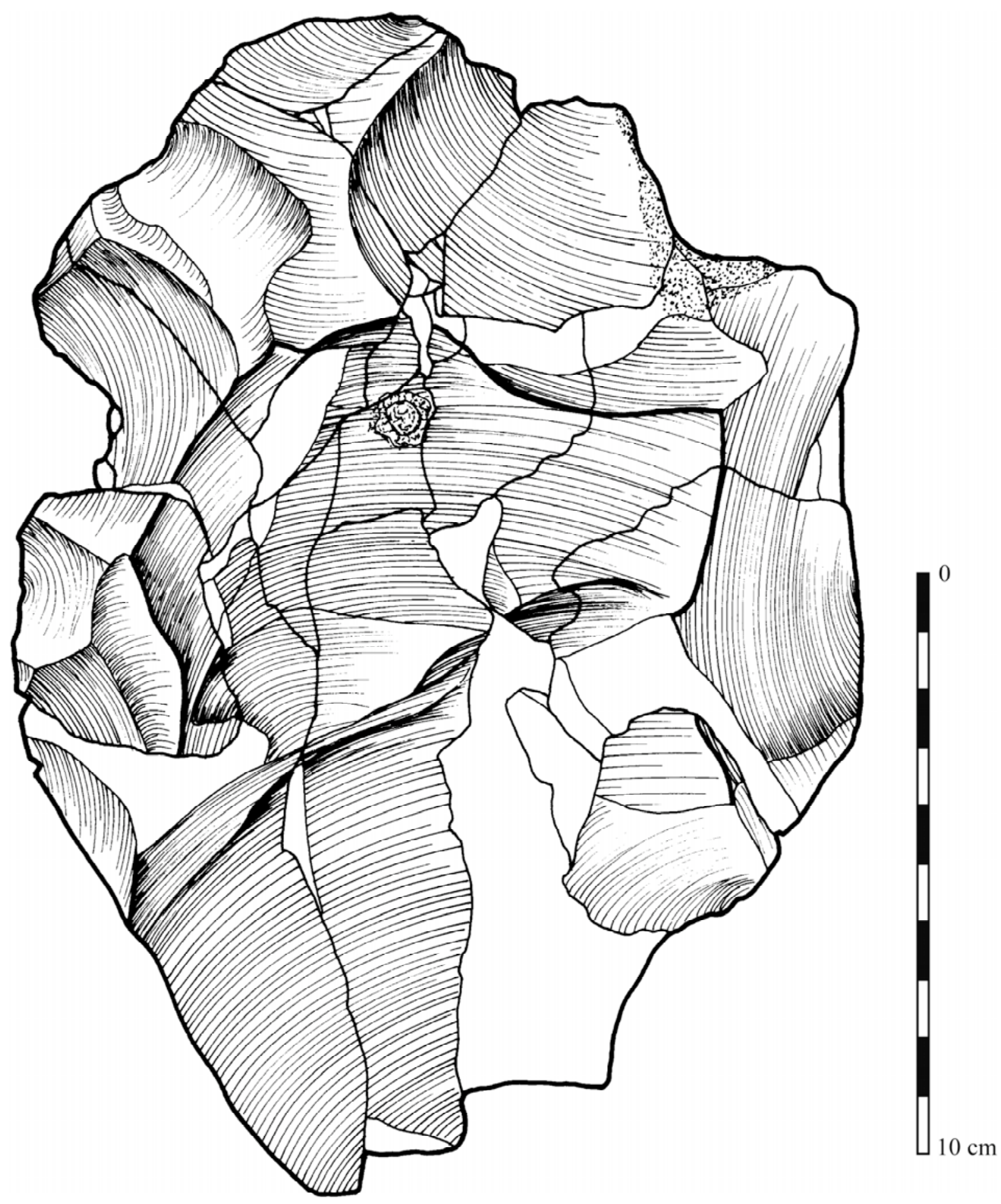

Figure 4 - Hermies-Le Champ Bruquette, remontage A98/deb91.

Figure 4 - Hermies-Le Champ Bruquette, refitting A98/deb91. 


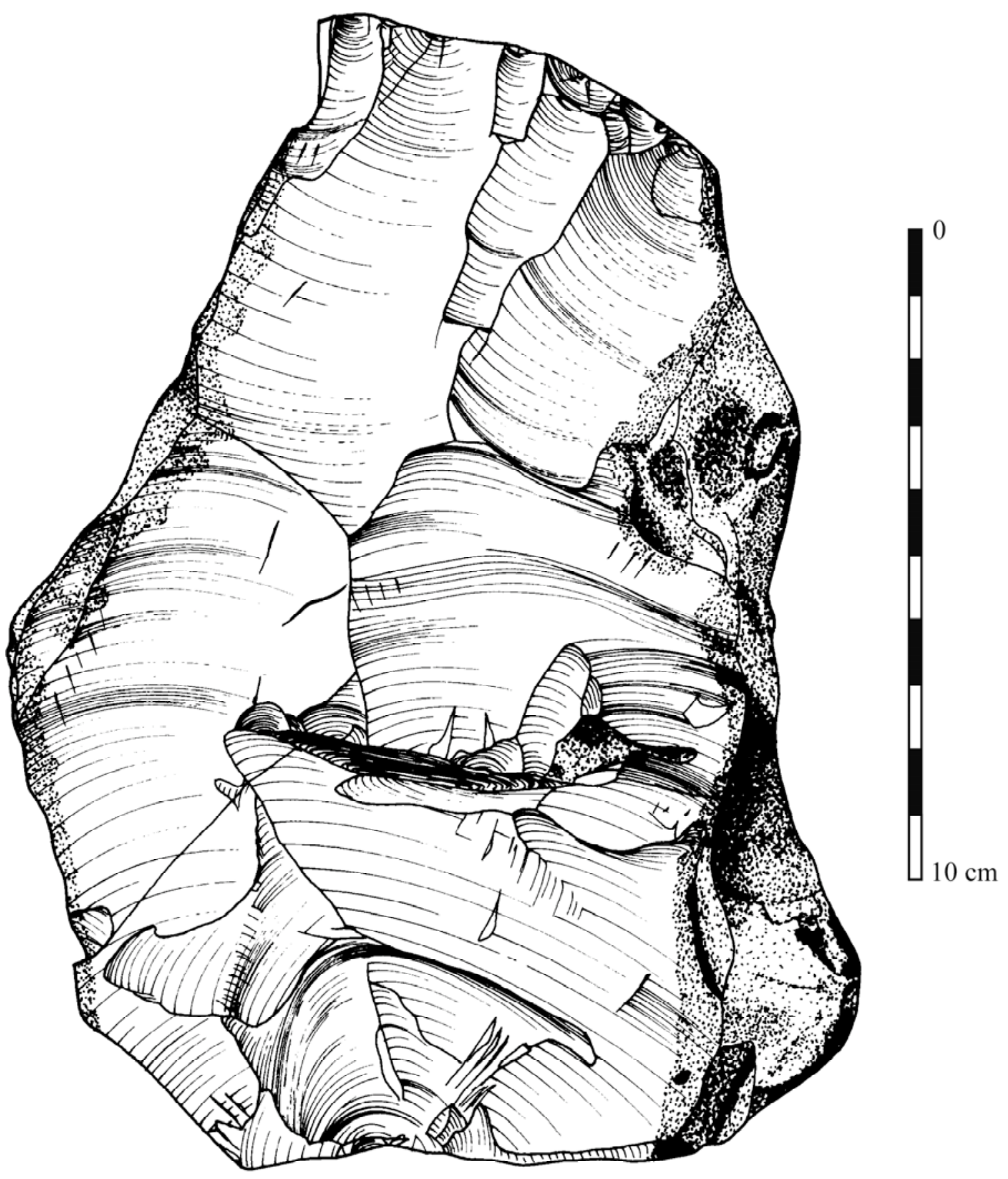

Figure 5 - Hermies-Le Champ Bruquette, nucléus A98/10.

Figure 5 - Hermies-Le Champ Bruquette, Levallois core A98/10. 
ou moins étendue du pourtour, lorsqu'il s'agissait d'un bloc de forme plus ou moins lenticulaire, ou par enlèvements uni- ou bipolaires dans le grand axe du rognon, essentiellement aux dépens de la future surface Levallois, lorsqu'il s'agissait de rognons plus ou moins allongés : dans ce cas, le dégrossissage profitait souvent des aspérités qui fournissaient un plan de frappe adéquat. Lorsque le bloc présentait une forme suffisamment cylindrique, le plan de frappe utilisé pour la mise en forme de la surface de débitage était ouvert par un enlèvement orthogonal à l'allongement, tronquant une extrémité du rognon (il pouvait s'agir d'une surface de fracture naturelle). Dans le cas de blocs globuleux ou biscornus, la mise en forme présente un aspect intermédiaire, avec des enlèvements alternants périphériques exploitant de façon opportuniste les aspérités du rognon. Dans tous les cas, les enlèvements sur la future surface de plan de frappe restent marginaux : celle-ci demeure très largement corticale et peut conserver une protubérance marquée.

Sur 39 nucléus retrouvés dans le niveau a du Champ Bruquette (Pléniglaciaire inférieur weichsélien), 32 sont des nucléus Levallois à éclat préférentiel, 1 est de mode récurrent bipolaire, les autres présentent un débitage unipolaire. Les nucléus Levallois montrent l'enlèvement d'un éclat envahissant ovalaire ou subcirculaire sur une surface de débitage préparée par des enlèvements essentiellement centripètes (Masson et Vallin 1993) ; la surface de préparation du plan de frappe ne porte que quelques enlèvements périphériques, souvent limités aux extrémités proximale et distale du nucléus. Le plan de frappe est souvent facetté, mais assez sommairement. Dans le détail, la préparation de la surface Levallois montre un certain nombre de traits bien spécifiques, constants d'une séquence de réduction à l'autre et même d'un amas à l'autre (comme par exemple la fréquente dissymétrie dans la préparation des convexités latérales, un bord latéral -plutôt le gauche- présentant souvent des enlèvements allongés débordants, de direction parallèle à l'axe Levallois, alors que le bord latéral opposé présente plus fréquemment des enlèvements centripètes) (fig. 2) ; cette dissymétrie provoque souvent un outrepassage latéral de l'éclat préférentiel, dont on pourrait se demander s'il n'était pas recherché (fig. 3). Les remontages ont montré que le(s) tailleur(s) avai(en)t re-préparé la surface Levallois, chaque fois que c'était possible, pour obtenir un autre éclat préférentiel (fig. 4) ; cette séquence a été répétée jusqu'à quatre fois. Seuls deux ou trois nucléus relèvent d'un autre schéma opératoire : ils sont également préférentiels mais la surface Levallois est préparée par débitage bipolaire opposé dans l'axe longitudinal de blocs plutôt allongés (fig. 5).

Sur 30 nucléus Levallois entiers, la moyenne des dimensions donne $127 \times 111 \times 57 \mathrm{~mm}$, pour un poids moyen de $742 \mathrm{~g}$ (ces chiffres différent peu des médianes) ; le nucléus le plus volumineux mesure $178 \times 113 \times 99 \mathrm{~mm}$ et pèse $1.560 \mathrm{~g}$; le plus petit mesure $93 \times 84 \times 54 \mathrm{~mm}$ pour un poids de $335 \mathrm{~g}$.

Dans les niveaux appartenant à la phase principale d'occupation du Tio Marché (Pléniglaciaire moyen weichsélien), le nombre total de nucléus (blocs testés exclus) s'élève à près de 120. La quasi-totalité des nucléus sont
Levallois ; ceux-ci relèvent le plus souvent d'un débitage linéal de préparation centripète et sont alors semblables à ceux du Champ Bruquette, d'autres présentent une préparation bipolaire opposée ou unipolaire convergente ; le reste des nucléus Levallois est représenté par des nucléus récurrents, unipolaires convergents, bipolaires ou centripètes (dont une bonne partie provient du recyclage de nucléus Levallois de mode linéal). Beaucoup de nucléus de mode linéal présentent une forte ressemblance qui permettrait de les regrouper par catégories morphologiques (fig. 6, 7 et 8) ; cela peut tenir à l'envahissement du négatif de l'éclat préférentiel, à la dissymétrie des profils longitudinaux et transversaux, aux modalités de préparation de la surface Levallois (nombre et disposition des enlèvements), à l'utilisation d'éclats débordants pour l'élaboration des convexités latérales, au débordement de l'éclat préférentiel lui-même (comme au Champ Bruquette), etc. Sur 68 nucléus Levallois entiers, la moyenne des dimensions donne $114 \times 102 \times 50 \mathrm{~mm}$, pour un poids moyen de $597 \mathrm{~g}$ (ces chiffres différent peu des médianes), soit une légère diminution par rapport au niveau a du Champ Bruquette. Malgré l'homogénéité technique globale, la différence est importante entre le plus gros nucléus Levallois, qui mesure $175 \times 117 \times 75 \mathrm{~mm}$ et pèse $1492 \mathrm{~g}$ (à peu près les mêmes dimensions que le plus gros nucléus du Champ Bruquette-niveau a) et le plus petit, dont les dimensions sont de $50 \times 47 \times 30 \mathrm{~mm}$ pour un poids de $52 \mathrm{~g}$. La grande cohérence d'ensemble des schémas opératoires s'accompagne donc d'une certaine variabilité, au moins du point de vue morphométrique ; il conviendra de rechercher, dans la gamme des produits finis, la trace de ces différences.

Plusieurs remarques importantes doivent cependant être prises en compte:

- la répétition, sur le même nucléus, de plusieurs séquences de production d'éclat préférentiel (tant sur le site du Champ Bruquette que sur celui du Tio Marché) ; théoriquement, la re-préparation des surfaces devrait amener à une réduction graduelle et proportionnelle du nucléus et des éclats Levallois produits ; en fait les remontages ont suscité des constatations inverses pour les produits finis, l'éclat de deuxième ordre étant souvent plus envahissant que l'éclat de premier ordre (fig. 9, 10, 11, 12) ;

- la ramification de la chaîne opératoire, par recyclage des fragments de bloc ou de fragments de nucléus, générés (accidentellement ou non) à un moment ou à un autre de la séquence de réduction ; ce processus, contrairement à ce qu'on a pu constater au Champ Bruquette, paraît assez fréquent au Tio Marché. Dans certains cas, il semble qu'il y ait eu un traitement différencié, hiérarchisé, entre les fragments issus du même rognon : le fragment principal était réservé à un schéma opératoire Levallois, alors que le(s) fragment(s) secondaire(s) participai(en)t à un débitage de type opportuniste, tel que le débitage centripète d'éclats larges et réfléchis ou le débitage semi-tournant d'éclats allongés aux dépens d'un bord ;

- la manifestation de niveaux de savoir-faire très différents, qui conduisent à évoquer l'hypothèse de l'activité de jeunes, en phase d'acquisition des gestes techniques, explication soutenue par l'existence de débitages sommaires, qui présentent une répartition spatiale périphérique par rapport aux postes de débitage (Vallin et Masson 2004). 

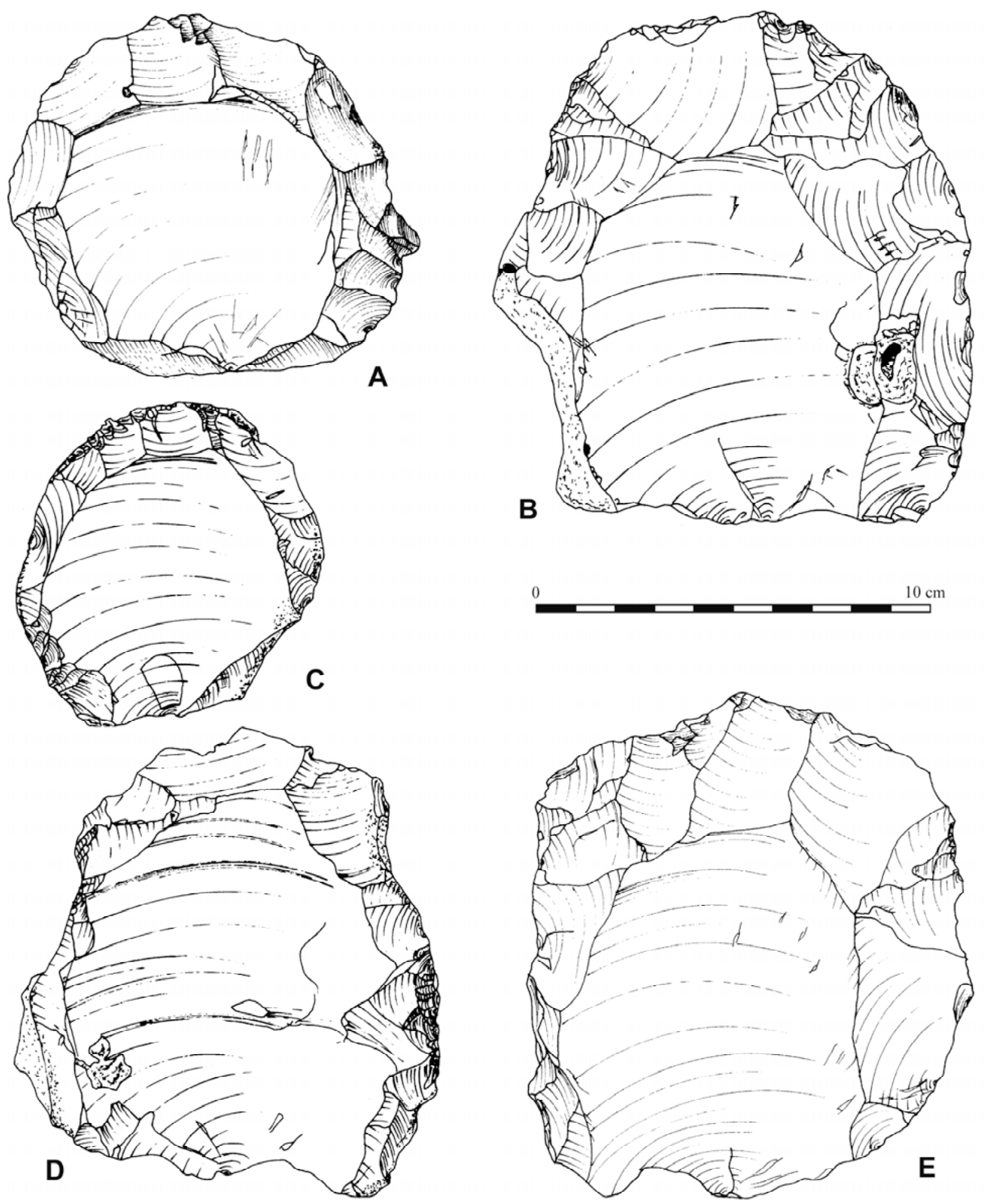

Figure 6 - Parenté entre nucléus Levallois d'Hermies-Le Tio Marché : A-N17/17 et C-T12/45, B-O6/1 et D- R12/108 et E-P1/3. Figure 6 - Similarity of Levallois cores from Hermies-Le Tio Marché : A-N17/17 and C-T12/45, B-O6/1 and D- R12/108 and EP1/3. 

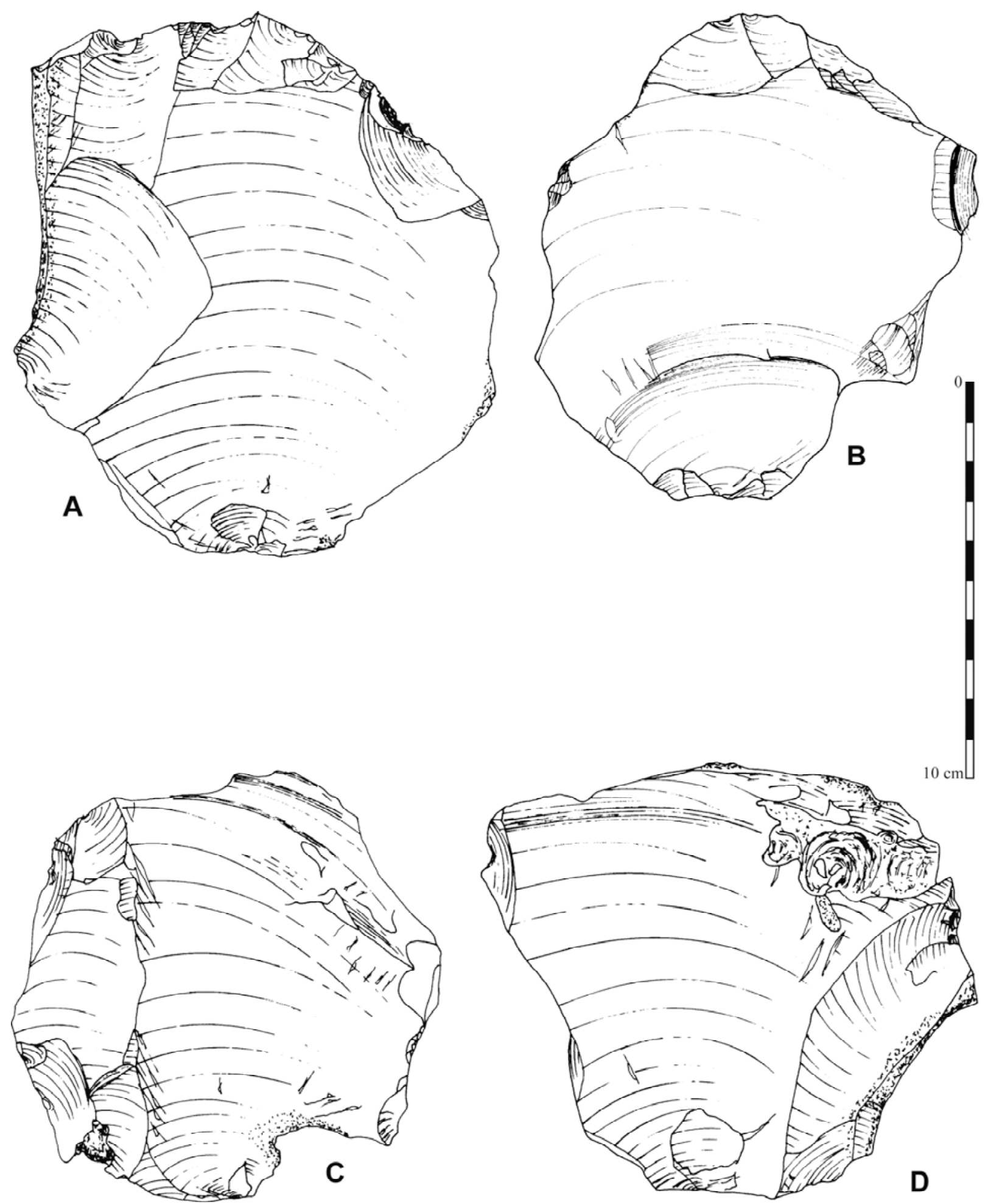

Figure 7 - Parenté entre nucléus Levallois d'Hermies-Le Tio Marché : A-F22/déb9 et B-P8/8, C-G21/4 et D-N24/1.

Figure 7 - Similarity of Levallois cores from Hermies-Le Tio Marché : A-F22/déb9 and B-P8/8, C-G21/4 and D-N24/1. 

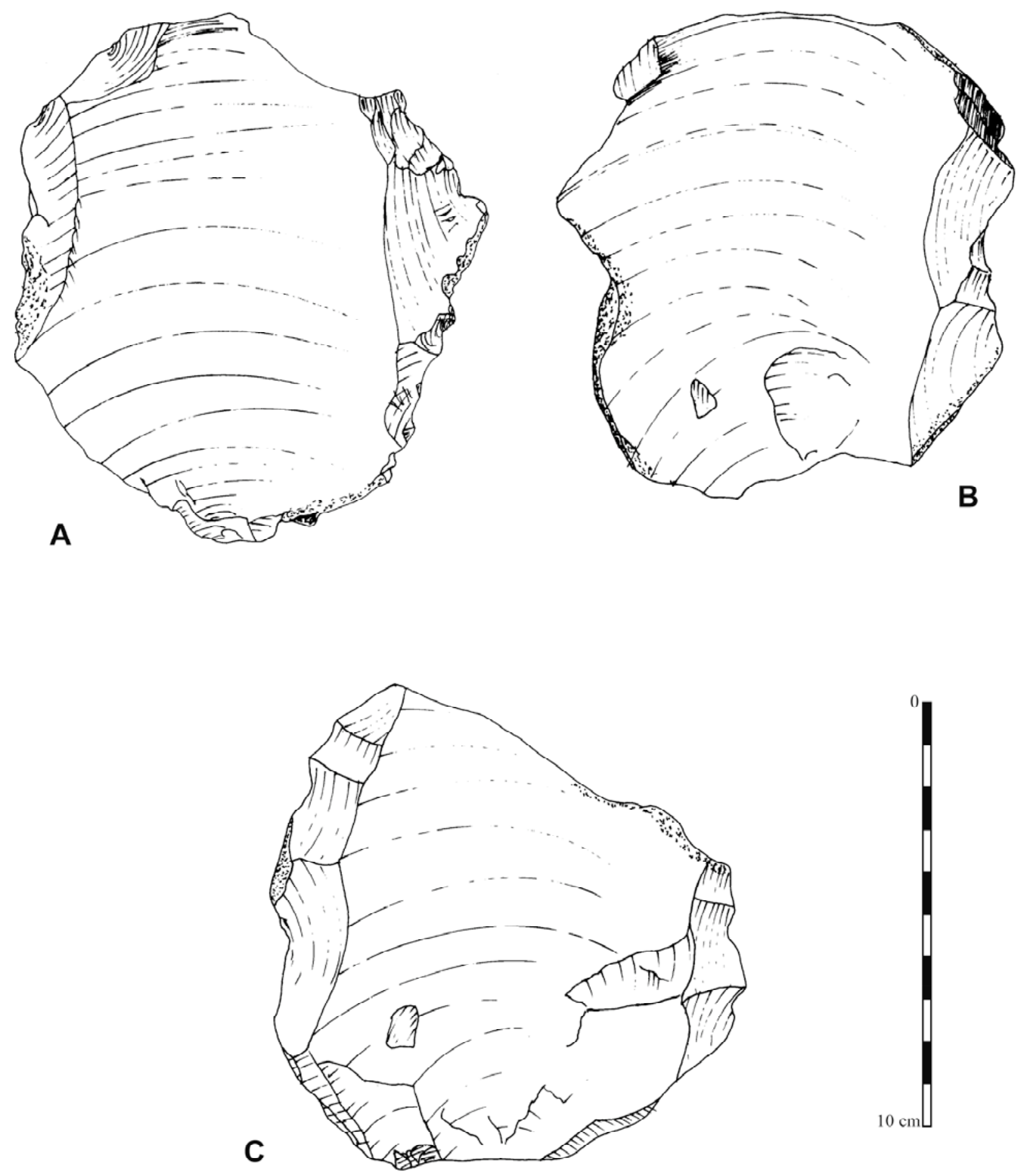

Figure 8 - Parenté entre nucléus Levallois d'Hermies-Le Tio Marché : A-R11/8 et B-M18/20 et C-R11/3.

Figure 8 - Similarity of Levallois cores from Hermies-Le Tio Marché : A-R11/8 and B-M18/20 and C-R11/3. 

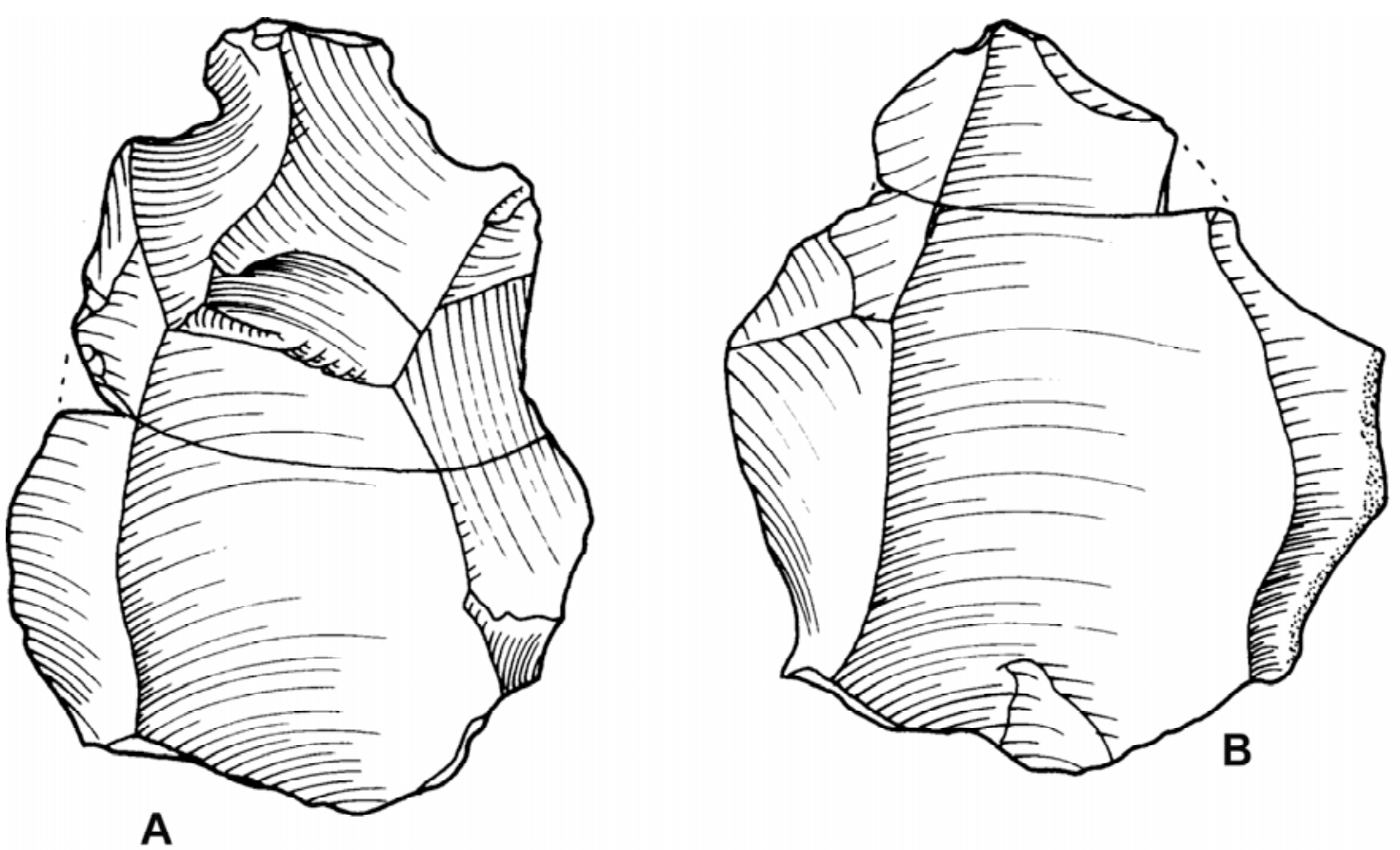

$10 \mathrm{~cm}$
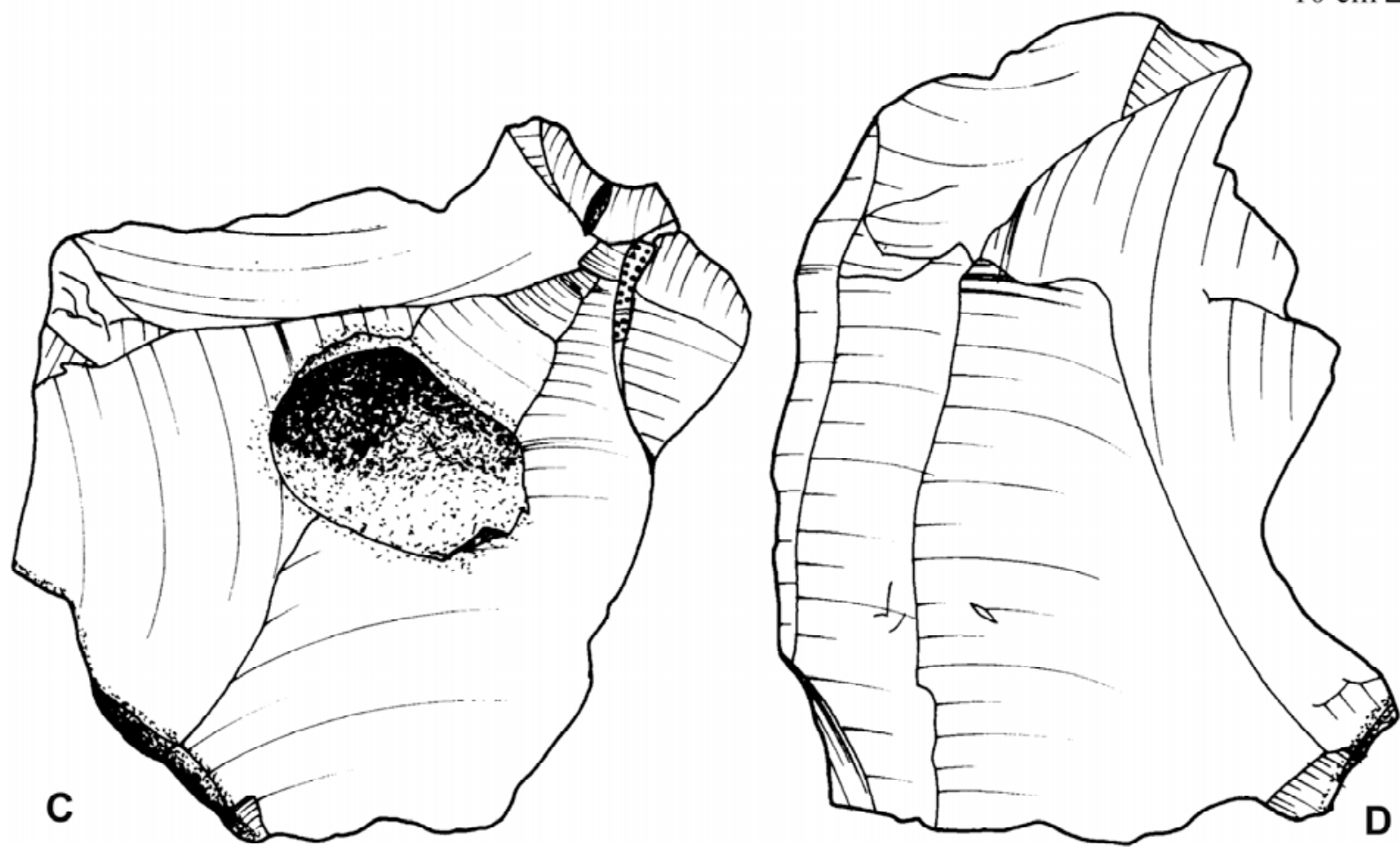

Figure 9 - Comparaison entre éclats préférentiels successifs d'Hermies-Le Champ Bruquette : A-AG67/1 et B-AG67/2, CA98/38 et $D-A 98 / 37$.

Figure 9 - Comparison between successive preferential flakes from Hermies-Le Champ Bruquette : A-AG67/1 and B-AG67/2, $C-A 98 / 38$ and $D-A 98 / 37$. 


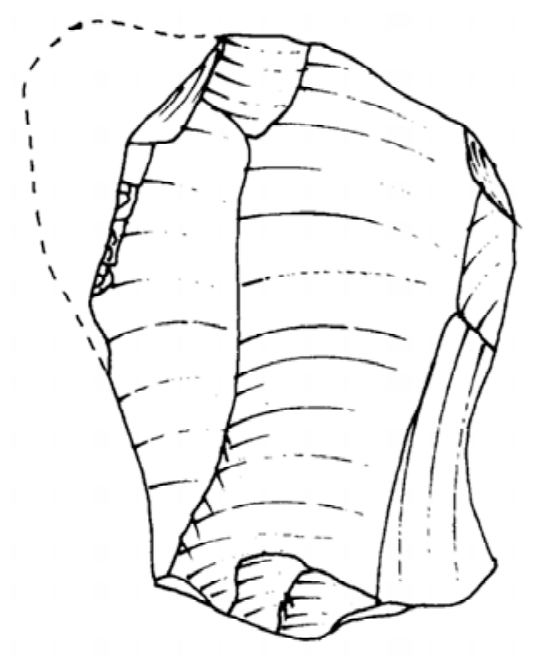

A
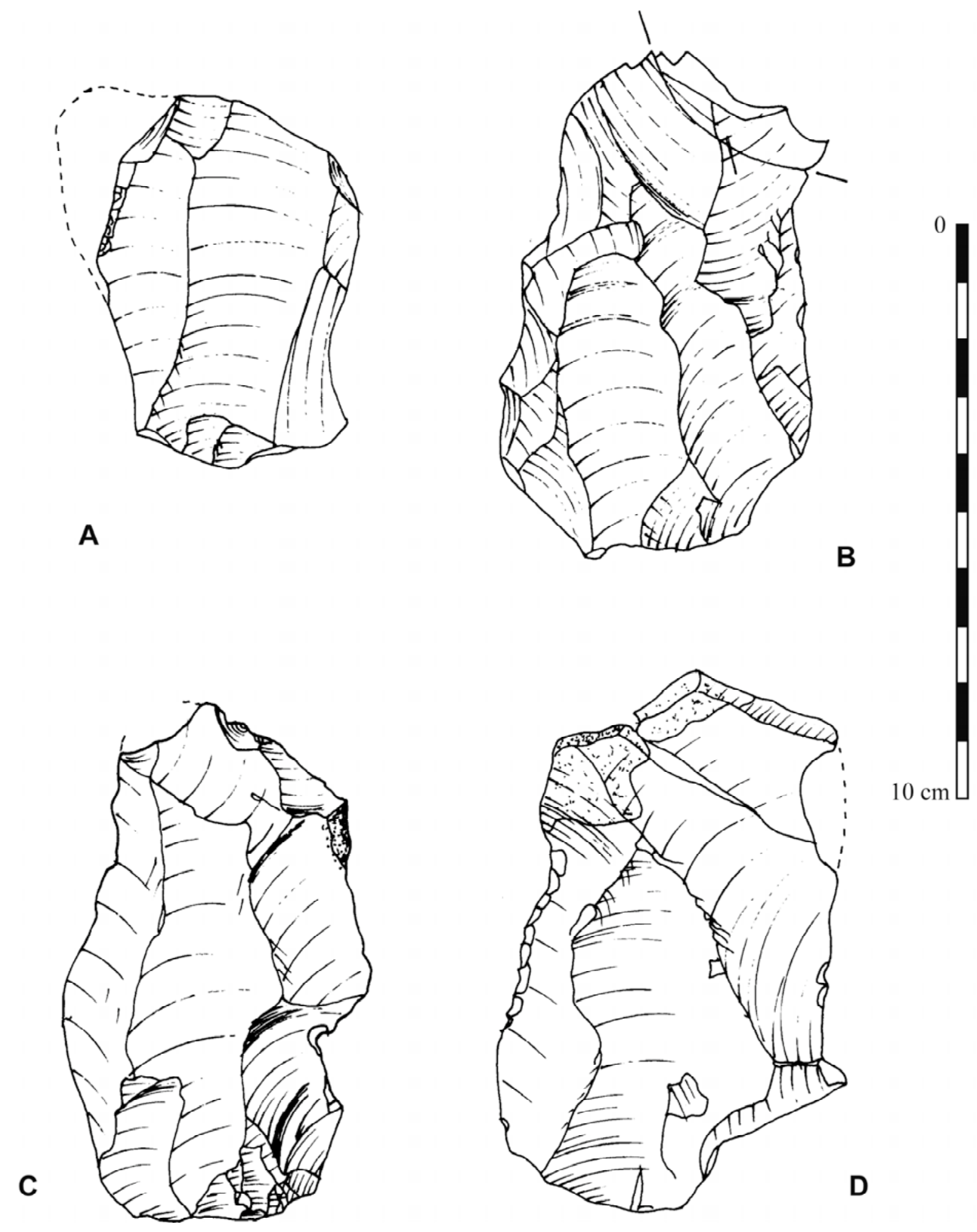

Figure 10 - Comparaison entre éclats préférentiels successifs d'Hermies-Le Tio Marché : A-P21/75 et B-P21/15, C-T17/11 et D-T17/7.

Figure 10 - Comparison between successive preferential flakes from Hermies-Le Tio Marché : A-P21/75 and B-P21/15, CT17/11 and D-T17/7. 


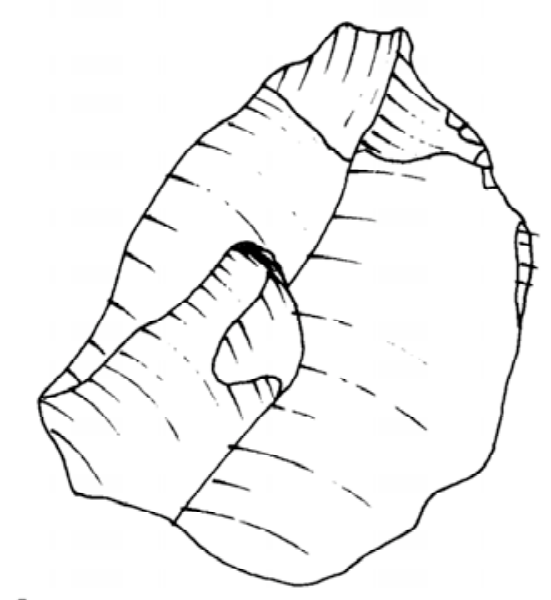

A
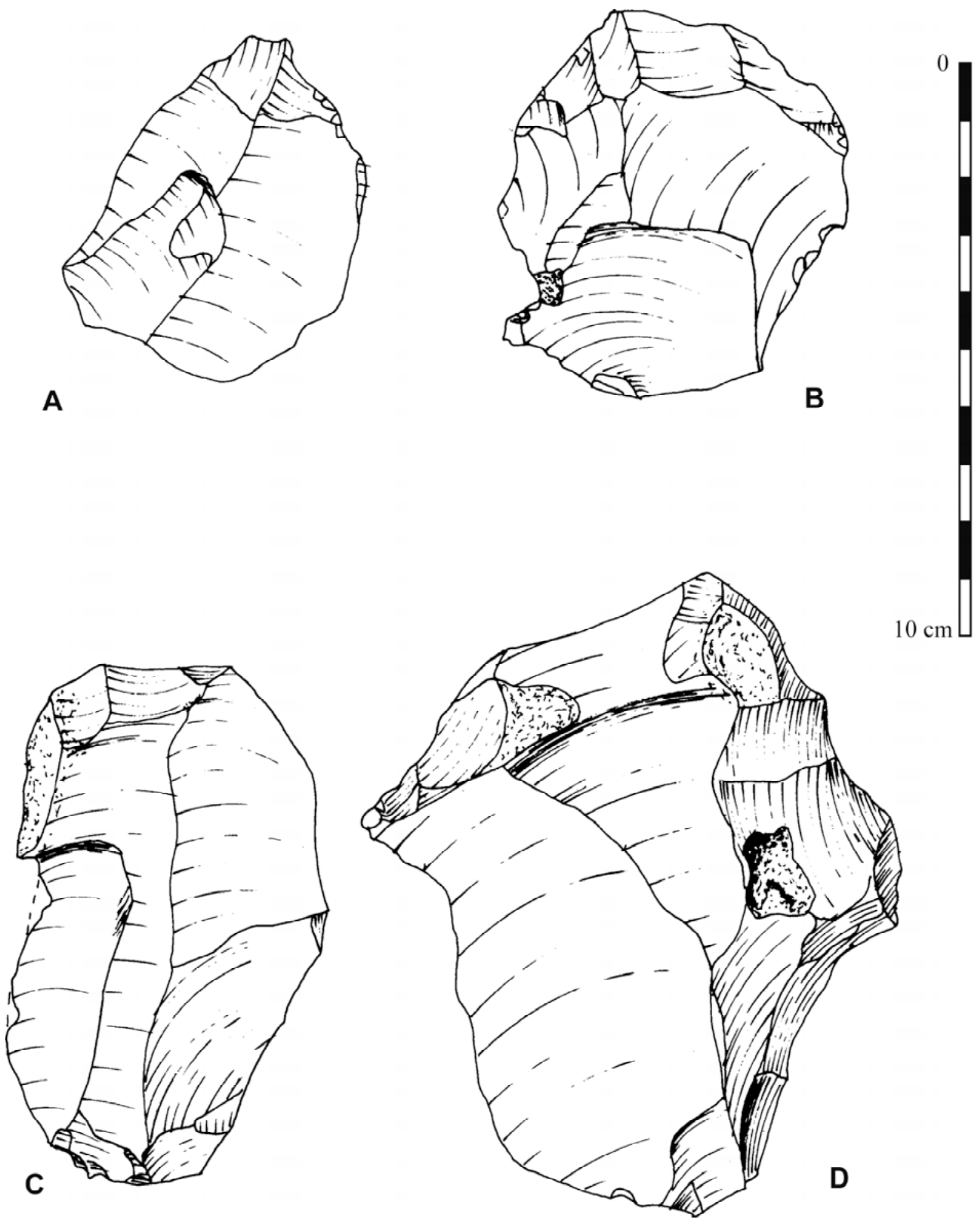

Figure 11 - Comparaison entre éclats préférentiels successifs d'Hermies-Le Tio Marché : A-U17/30 et B-U17/10, C-F18/30 et $D-F 18 / 9$.

Figure 11 - Comparison between successive preferential flakes from Hermies-Le Tio Marché : A-U17/30 and B-U17/10, CF18/30 and D-F18/9. 

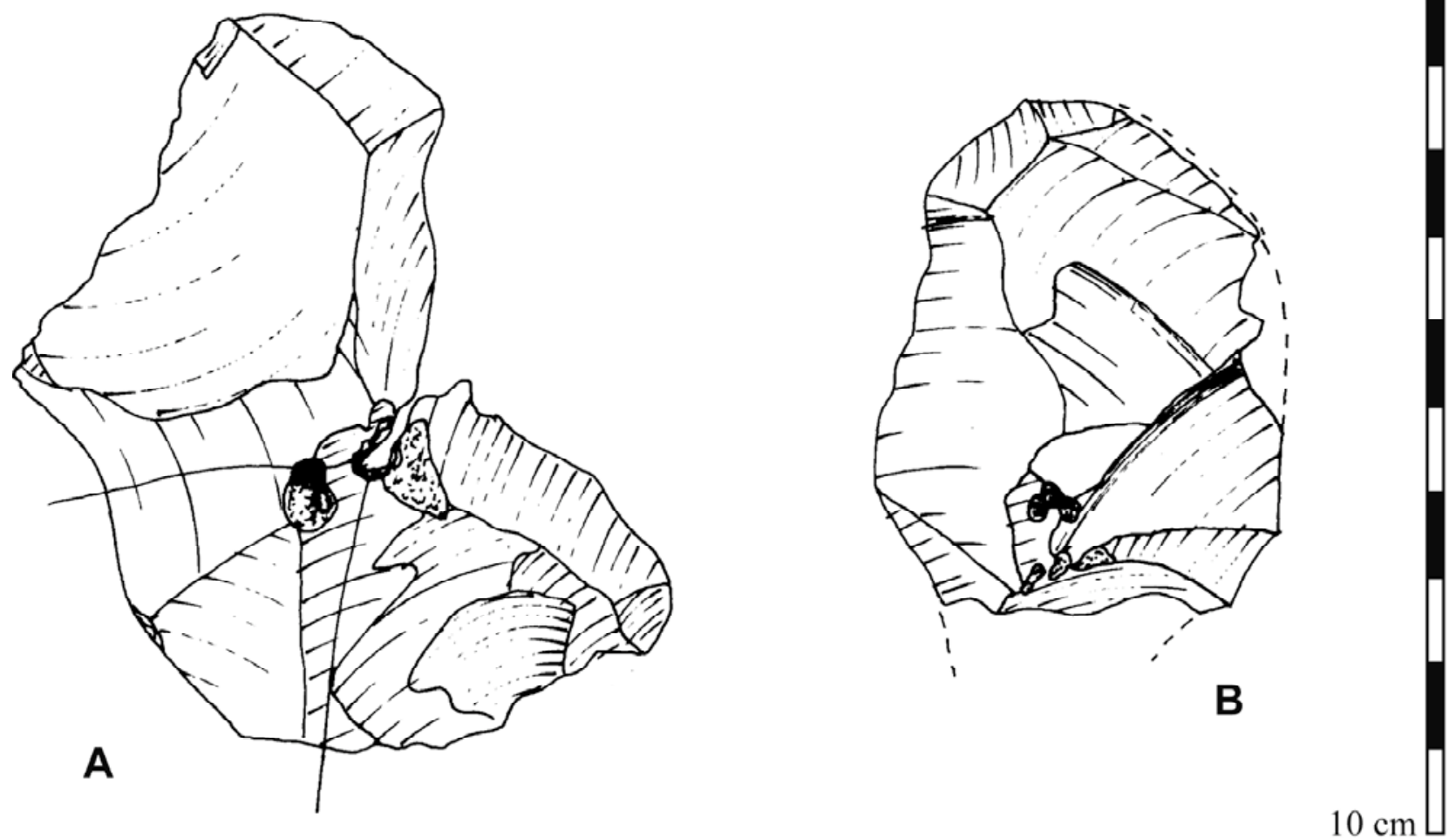

Figure 12 - Comparaison entre éclats préférentiels successifs d'Hermies-Le Tio Marché : A-Q5/91 (cassé en trois lors du débitage) et $B-Q 5 / 72$.

Figure 12 - Comparison between successive preferential flakes from Hermies-Le Tio Marché : A-Q5/91 (which was broken in three pieces during the knapping) and B-Q5/72.

Ces débitages opportunistes ou sommaires sont importants à pressentir car ils peuvent brouiller l'image et l'appréciation de l'objectif principal de la production.

\section{3 - Standardisation des produits}

Peut-on mettre en évidence une intention formelle des tailleurs d'Hermies, qui se traduirait par une homogénéité morphologique ou dimensionnelle des éclats préférentiels ou par un classement en gabarits différenciés ou bien, au contraire, y a t-il une production plutôt aléatoire, la standardisation (éventuelle) n'intervenant qu'au niveau du choix des pièces utilisées ? En corollaire, les éclats Levallois résultent-ils entièrement d'une production sur place, ou bien y a t-il coexistence de produits de type " domestique " et de produits "importés " comme cela a pu être évoqué sur certains sites moustériens à propos de l'outillage retouché ? Nous nous sommes attachés à la recherche d'éventuelles constantes de production par l'examen de la répartition des populations du Champ Bruquette et du Tio Marché pour chaque variable.
Un certain nombre de corrélations peuvent être mises en évidence entre les caractères morphotechniques et fonctionnels des bords. Certaines relèvent plutôt de contraintes liées à la mécanique du détachement au percuteur dur : par exemple, on constate que l'angulation du bord diminue presque toujours de la partie proximale à la partie distale, ce qui découle en l'occurence de l'amincissement des éclats depuis le bulbe jusqu'au bout (tabl. 3), particulièrement sensible dans le cas d'une percussion au percuteur de pierre dure dont le point d'impact se situe bien en retrait du bord du plan de frappe. La moyenne de l'épaisseur maximale du talon se situe, par exemple, à 14,1 $\mathrm{mm}$ pour les éclats préférentiels du Champ Bruquette (avec un écart-type de 4,5 mm).

Les bords proximaux présentent donc une angulation moyenne oblique, assez comparable entre le bord gauche et le bord droit (respectivement $43^{\circ}$ et $44^{\circ}$ ) ; cet angle moyen est supérieur à celui des bords distaux $\left(38^{\circ}\right.$ pour le bord gauche distal, $36^{\circ} 30$ pour le bord distal et $37^{\circ}$ pour le bord droit distal). II existe une corrélation entre la délinéation du bord et son angulation, dans la mesure où les 

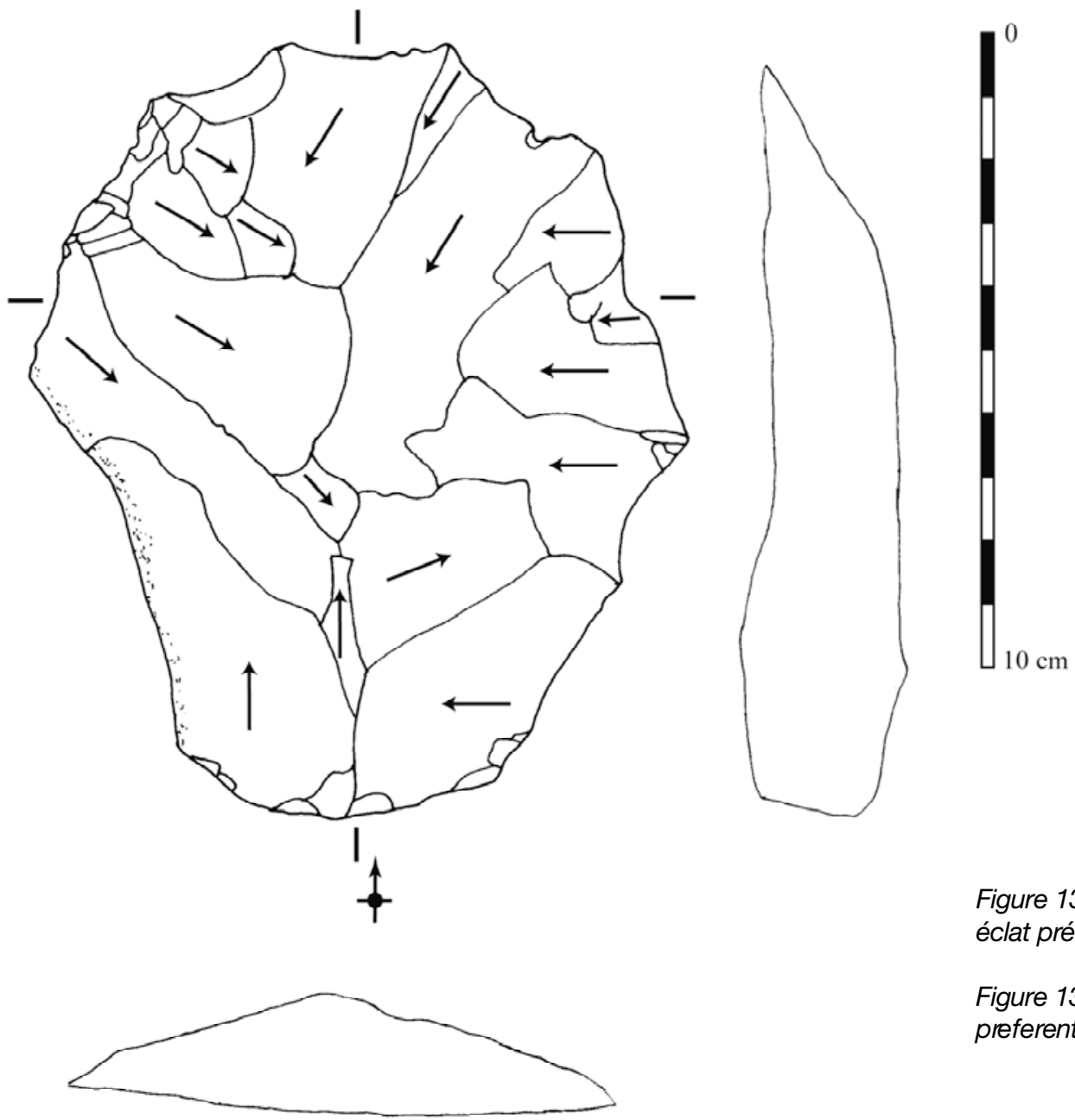

Figure 13 - Hermies-Le Tio Marché, éclat préférentiel $P 21 / 116$.

Figure 13 - Hermies-Le Tio Marché, preferential flake P21/116.

\begin{tabular}{|l|c|l|c|l|c|}
\hline Bord & $\begin{array}{l}\text { gauche } \\
\text { proximal }\end{array}$ & $\begin{array}{l}\text { gauche } \\
\text { distal }\end{array}$ & distal & $\begin{array}{l}\text { droit } \\
\text { distal }\end{array}$ & $\begin{array}{l}\text { droit } \\
\text { proximal }\end{array}$ \\
\hline $\begin{array}{l}\text { nombre de bords non } \\
\text { corticaux et sans défaut }\end{array}$ & 90 & 100 & 75 & 102 & 95 \\
\hline médiane des angles & $41^{\circ} 30$ & $36^{\circ}$ & $32^{\circ}$ & $35^{\circ}$ & $42^{\circ}$ \\
\hline moyenne des angles & $43^{\circ}$ & $38^{\circ}$ & $36^{\circ} 30$ & $37^{\circ}$ & $44^{\circ}$ \\
\hline écart-type & 12,39 & 10,43 & 12,27 & 10,5 & 12,42 \\
\hline $\begin{array}{l}\text { médiane des longueurs } \\
\text { de tranchant }\end{array}$ & $38 \mathrm{~mm}$ & $41,5 \mathrm{~mm}$ & $69 \mathrm{~mm}$ & $38 \mathrm{~mm}$ & $38 \mathrm{~mm}$ \\
\hline $\begin{array}{l}\text { moyenne des longueurs } \\
\text { de tranchant }\end{array}$ & $39,2 \mathrm{~mm}$ & $42,5 \mathrm{~mm}$ & $69,4 \mathrm{~mm}$ & $40,1 \mathrm{~mm}$ & $39 \mathrm{~mm}$ \\
\hline écart-type & 15,39 & 17,08 & 30,46 & 13,85 & 12,83 \\
\hline
\end{tabular}

Tableau 3 - Angulation moyenne et longueur moyenne de tranchant utile par segment de bord des éclats préférentiels d'Hermies mesurés (tous niveaux confondus, $n=133$ ).

Table 3 - Angle average and length average of cutting edge by part of side on preferential flakes from Hermies (all levels taken into account, $n=133$ ). 


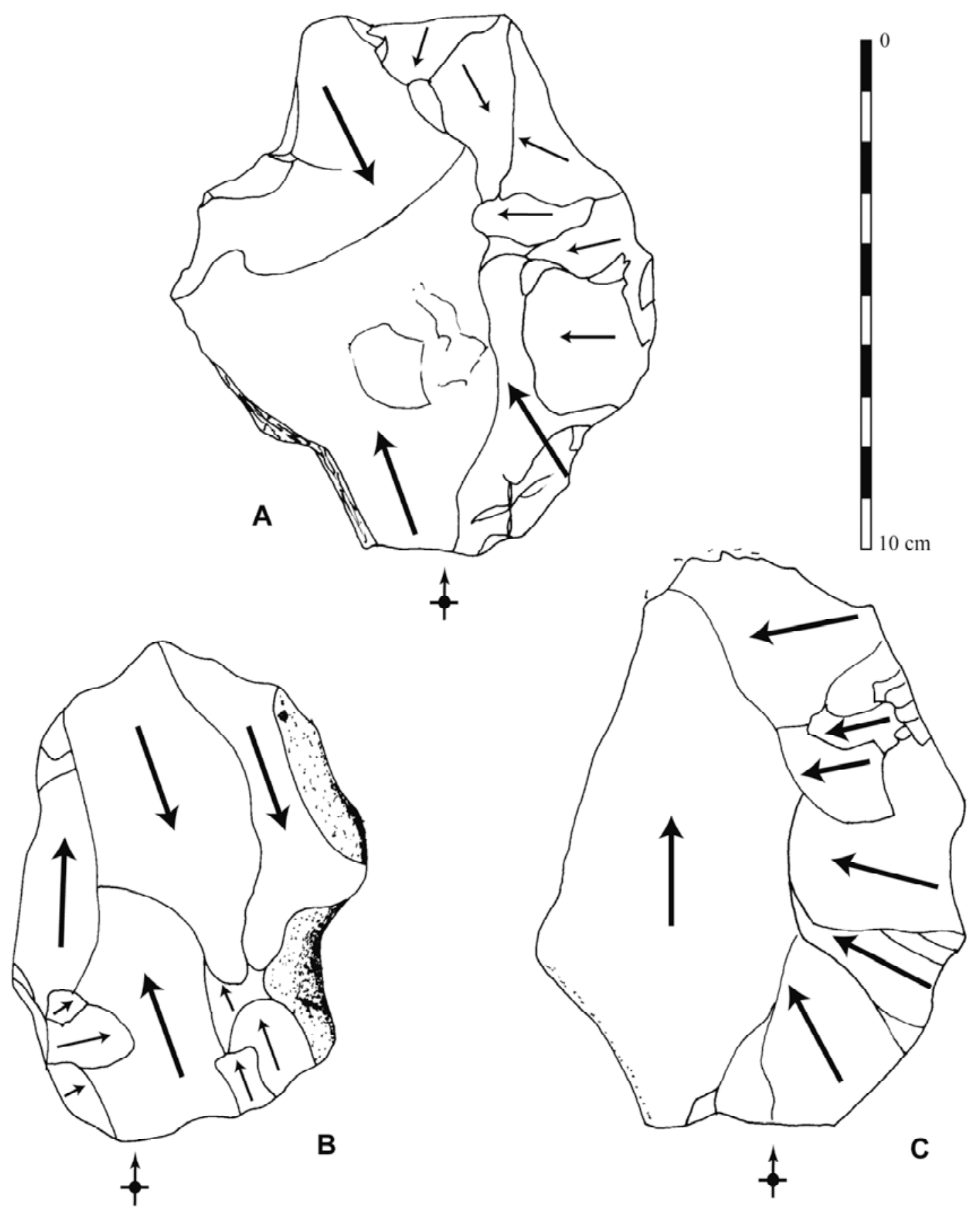

Figure 14 - Eclats Levallois débordants à gauche (A-AD82/3 du Champ Bruquette), éclats Levallois à bord rectiligne (B-E18/1 et C-F19/5 du Tio Marché).

Figure 14 - Levallois éclats débordants (A-AD82/3 from Champ Bruquette), Levallois flake with straight edge (B-E18/1 and CF19/5 from Tio Marché). 


\begin{tabular}{|c|c|c|c|c|c|c|c|c|}
\hline Bord & $\begin{array}{l}\text { gauche } \\
\text { proximal }\end{array}$ & $\begin{array}{l}\text { gauche } \\
\text { distal }\end{array}$ & distal & \begin{tabular}{|l|} 
droit \\
distal
\end{tabular} & $\begin{array}{l}\text { droit } \\
\text { proximal }\end{array}$ & Total Hermies & Total CB & Total TM \\
\hline convexe & 41 & 35 & 44 & 38 & 33 & 191 & 29 & 162 \\
\hline rectiligne & 36 & 33 & 10 & 44 & 51 & 174 & 38 & 136 \\
\hline concave & 27 & 29 & 1 & 22 & 24 & 103 & 21 & 82 \\
\hline sinueux & 14 & 20 & 24 & 16 & 10 & 84 & 28 & 56 \\
\hline festonné & 13 & 15 & 29 & 10 & 15 & 82 & 17 & 65 \\
\hline arc brisé & 2 & 1 & 25 & 3 & 0 & 31 & 7 & 24 \\
\hline \multicolumn{9}{|l|}{ dont : } \\
\hline cortical & 21 & 16 & 10 & 17 & 15 & 79 & 17 & 62 \\
\hline défaut & 34 & 24 & 57 & 24 & 29 & 168 & 45 & 123 \\
\hline modifié & 21 & 26 & 37 & 19 & 24 & 127 & 19 & 108 \\
\hline utilisé & 5 & 6 & 7 & 14 & 10 & 42 & 4 & 38 \\
\hline
\end{tabular}

Tableau 4 - Typologie des segments de bord des éclats préférentiels d'Hermies mesurés (tous niveaux confondus, $n=133$ ). $C B=$ Champ Bruquette, $T M=$ Tio Marché.

Table 4 - Typology of sides on preferential flakes from Hermies (all levels taken into account, $n=133$ ). CB = Champ Bruquette, TM = Tio Marché

bords concaves présentent les angles les plus ouverts (médiane : $46^{\circ}$ ), suivis par les bords sinueux et festonnés, puis rectilignes (médianes respectives : $42^{\circ} 30,42^{\circ}$ et $41^{\circ}$ ), tandis que les bords convexes possèdent les angles les plus fermés (médiane : $39^{\circ}$ ).

La délinéation des bords est évidemment sous la dépendance de la préparation de la surface Levallois (Boëda 1994) : des enlèvements prédéterminants centripètes auront tendance à préfigurer un bord globalement convexe, si leur inclinaison est rasante, ou un bord festonné (c'est-à-dire présentant des concavités contigües), si la surface de fracture de l'éclat préférentiel recoupe les contre-bulbes prononcés de ces enlèvements (fig. 13) ; par contre, un bord rectiligne continu, parallèle à l'axe longitudinal et d'angle semi-abrupt, sera généré par un enlèvement prédéterminant débordant latéralement, détaché parallèlement à l'axe Levallois. On retrouve sur les éclats, à cet égard, les mêmes types de préparation des surfaces Levallois que sur les nucléus, dans les mêmes proportions (fig. 14).

Le découpage du pourtour des éclats en segments de bord pour les nécessités de l'analyse informatique (en termes de présence/absence) présente le double inconvénient de biaiser la perception de la symétrie des éclats et d'interdire d'appréhender leurs côtés dans leur globalité : ainsi la continuité ou la discontinuité d'un bord, de la partie proximale à la partie distale, ne pourra pas être reconnue. Cela explique peut-être l'image de relative symétrie qui se dégage de la répartition typologique moyenne des bords, malgré les observations effectuées sur le débordement fréquent des éclats. Le type de bord le plus fréquent (tabl. 4) est le bord convexe (191 cas), surtout présent en bout (en raison d'une préparation centripète de la convexité distale) et représenté de façon à peu près identique sur les autres segments du pourtour. Le bord rectiligne est presque aussi fréquent (174 cas), plus souvent sur le bord droit que sur le bord gauche, mais il concerne rarement le bout. Viennent ensuite le bord concave (103 cas), un peu plus présent sur le bord gauche mais quasiment absent en bout, puis le bord sinueux (84 cas) et le bord festonné (82 cas), plus fréquents sur les bords distaux que proximaux. L'arc brisé concerne presqu'uniquement le bord distal. C'est surtout le bord distal qui peut être affecté par un défaut (57 cas), en raison des outrepassages et rebroussements fréquents (qui concernent respectivement 24 et 50 objets), alors que c'est le bord proximal gauche qui est le plus souvent cortical. Ces observations portent sur l'ensemble des éclats préférentiels, toutefois il n'y a guère de différences entre les populations du Champ Bruquette et du Tio Marché, hormis le nombre relativement plus élevé de bords sinueux sur les éclats Levallois du Champ Bruquette (cf. infra).

La classification dimensionnelle des produits coïncide avec celle des nucléus. L'examen de l'ensemble des éclats préférentiels montre en effet un large éventail de dimensions, allant d'éclats assez petits $(58 \times 46 \times 10 \mathrm{~mm}$ pour l'éclat P6/83, fig. 15) à très grands $(166 \times 126 \times 39$ mm pour l'éclat R23/1, fig. 16) soit un rapport de 1 à 3 , avec des poids respectifs de 23 et $750 \mathrm{~g}$. Les mini-éclats Levallois, qui auraient pu provenir des plus petits nucléus du Tio Marché (mesurant moins de $6 \mathrm{~cm}$ de longueur), sont cependant absents (d'une manière plus générale, aucun remontage n'a été effectué sur ces mini-nucléus). On remarque que, sur les 14 éclats inférieurs à $70 \mathrm{~mm}$ de longueur, 10 sont rebroussés et ne traduisent probablement pas l'intention du tailleur. A l'autre extrémité de la gamme, sur 13 éclats dont la longueur excède ou égale $130 \mathrm{~mm}$, on constate que cinq sont outrepassés. Le périmètre des éclats (talon exclu) est un moyen de mesure plus proche des propriétés ergonomiques du support considéré que la longueur maximum. Sur 103 individus mesurables, la moyenne est de $242 \mathrm{~mm}$ (médiane : $234 \mathrm{~mm}$, écart-type : $60,6)$, avec des extrêmes de 135 et $420 \mathrm{~mm}$; la distinction selon les niveaux montre peu de variations (tabl. 5).

La répartition par classes montre que plusieurs pics se 


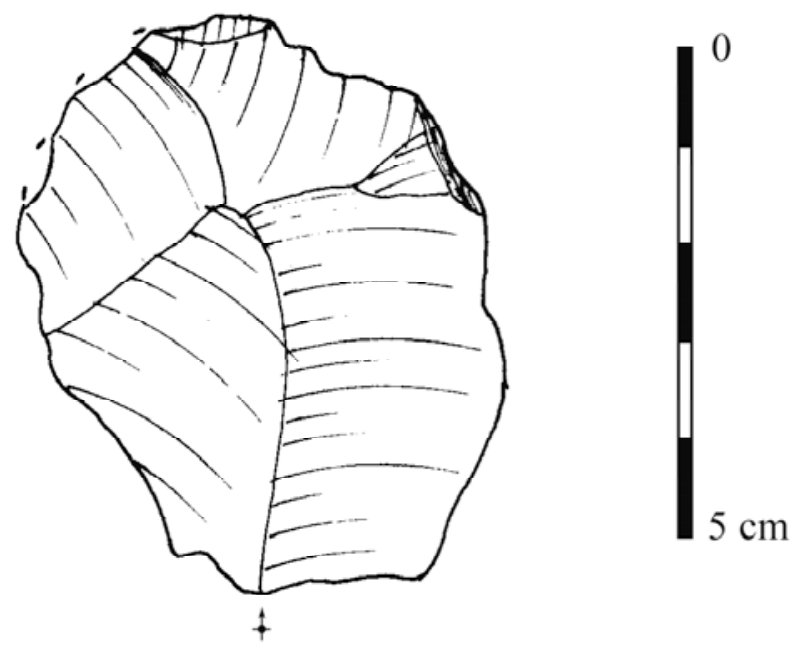

Figure 15 - Hermies-Le Tio Marché, éclat préférentiel $P 6 / 83$.

Figure 15 - Hermies-Le Tio Marché, preferential flake $P 6 / 83$.
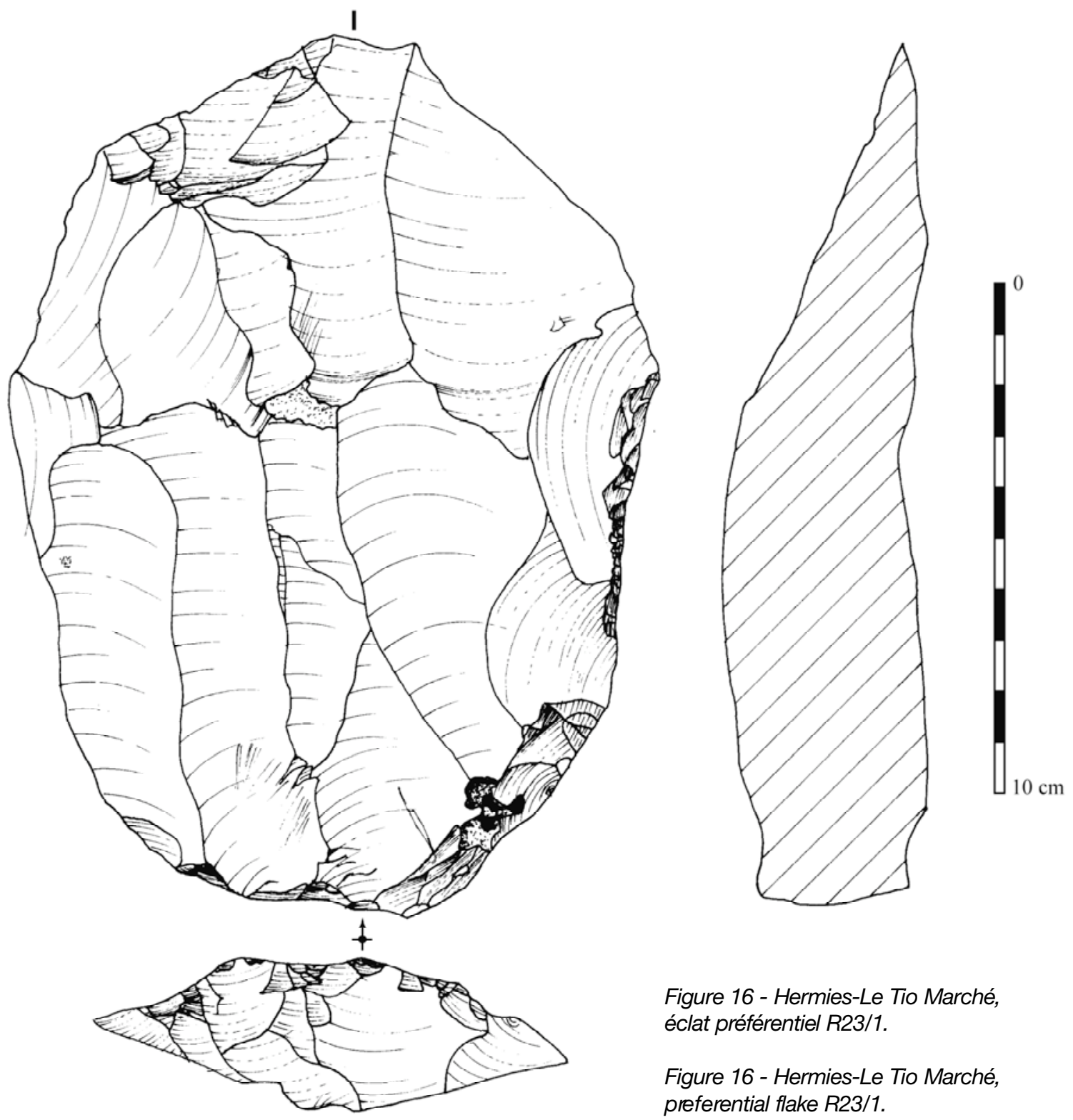

Figure 16 - Hermies-Le Tio Marché, éclat préférentiel R23/1.

Figure 16 - Hermies-Le Tio Marché, preferential flake $R 23 / 1$. 


\begin{tabular}{|c|c|c|c|c|c|}
\hline Niveau d'occupation & $\begin{array}{l}\text { Longueur moyenne } \\
\text { (éclats préférentiels } \\
\text { entiers) }\end{array}$ & $\begin{array}{l}\text { Allongement moyen } \\
\text { (éclats préférentiels } \\
\text { entiers) }\end{array}$ & $\begin{array}{l}\text { Poids moyen } \\
\text { (éclats préférentiels } \\
\text { entiers) }\end{array}$ & $\begin{array}{l}\text { Périmètre moyen } \\
\text { sans talon (éclats } \\
\text { préférentiels entiers) }\end{array}$ & Nombre \\
\hline $\begin{array}{l}\text { HTM, complexe } \\
\text { de Saint-Acheul }\end{array}$ & $\begin{array}{l}97,1 \mathrm{~mm} \\
\text { (écart-type : } 26,14 \text { ) }\end{array}$ & $\begin{array}{l}1,28 \\
\text { (écart-type : } 0,24 \text { ) }\end{array}$ & $\begin{array}{l}151,1 \mathrm{~g} \\
\text { (écart-type : 129,89) }\end{array}$ & $\begin{array}{l}246 \mathrm{~mm} \\
\text { (écart-type : 62,3) }\end{array}$ & 103 \\
\hline \begin{tabular}{|l} 
HCB, loess du \\
Pléniglaciaire \\
inférieur (niv. a)
\end{tabular} & $\begin{array}{l}94,4 \mathrm{~mm} \\
\text { (écart-type : 22,85) }\end{array}$ & $\begin{array}{l}1,22 \\
\text { (ècart-type : } 0,23 \text { ) }\end{array}$ & $\begin{array}{l}135,4 \mathrm{~g} \\
\text { (écart-type : 93,8) }\end{array}$ & $\begin{array}{l}240 \mathrm{~mm} \\
\text { (écart-type : 60,9) }\end{array}$ & 22 \\
\hline $\begin{array}{l}\text { HCB, surface } \\
\text { du cailloutis (niv. b) }\end{array}$ & $\begin{array}{l}91,4 \mathrm{~mm} \\
\text { (écart-type : } 12,20 \text { ) }\end{array}$ & $\begin{array}{l}1,28 \\
\text { (écart-type : 0,32) }\end{array}$ & $\begin{array}{l}138 \mathrm{~g} \\
\text { (ecart-type : 106,5) }\end{array}$ & $\begin{array}{l}215 \mathrm{~mm} \\
\text { (écart-type : } 60,6 \text { ) }\end{array}$ & 5 \\
\hline $\begin{array}{l}\text { HTM, surface } \\
\text { du cailloutis }\end{array}$ & $\begin{array}{l}100,3 \mathrm{~mm} \\
\text { (écart-type : } 21,87 \text { ) }\end{array}$ & $\begin{array}{l}1,44 \\
\text { (écart-type : } 0,17 \text { ) }\end{array}$ & $\begin{array}{l}130,5 \mathrm{~g} \\
\text { (écart-type : } 95,17\end{array}$ & $\begin{array}{l}261 \mathrm{~mm} \\
\text { (écart-type : } 56,2 \text { ) }\end{array}$ & 3 \\
\hline
\end{tabular}

Tableau 5 - Comparaison des dimensions moyennes des éclats préférentiels mesurés, par site et par niveau d'occupation. HTM = Hermies-Le Tio Marché, HCB = Hermies-Le Champ Bruquette.

Table 5 - Average sizes of the preferential flakes by site and occupation floor. HTM = Hermies-Le Tio Marché, HCB = HermiesLe Champ Bruquette.

dégagent : si la catégorie 205 à 279 mm regroupe $56 \%$ de l'effectif, une catégorie d'éclats plus petits (14\% de l'effectif entre 145 et $174 \mathrm{~mm}$, presque tous réfléchis) et une catégorie d'éclats plus grands (7\% de l'effectif entre 310 et $339 \mathrm{~mm}$ ) se distinguent, tandis que quelques éclats très grands (> $400 \mathrm{~mm}$ de périmètre, presque tous outrepassés) se détachent nettement du reste (fig. 17).

Afin de mesurer le degré d'efficacité de la méthode de débitage, en termes de production de tranchant utile, nous avons rapporté, pour chaque objet, le total de la longueur des bords tranchants au périmètre total de l'éclat (talon toujours exclu) (fig. 18). Les bords abrupts, corticaux, ou présentant un défaut sont évidemment exclus de la somme du tranchant utile. Sur 100 individus, la moyenne de tranchant utile est de $78 \%$ du pourtour (écart-type : 22, médiane : $85 \%$ ), ce qui est assez médiocre ; plus de la moitié des éclats présentent plus de $80 \%$ de tranchant utile, mais un cinquième de l'effectif est en-deçà de $60 \%$.

La classification des angles et des types de bord n'a pas permis de mettre en évidence une quelconque dissymétrie latérale (tabl. 3 et 4) ; par contre, la confrontation de la longueur de tranchant utile par côté, pour chaque éclat, montre un certain nombre de pièces à dos total ou partiel ; cette dissymétrie latérale n'apparaît pas dans les moyennes en raison de l'absence de latérisation préférentielle des pièces à dos cortical ou de préparation : le nombre d'éclats présen-

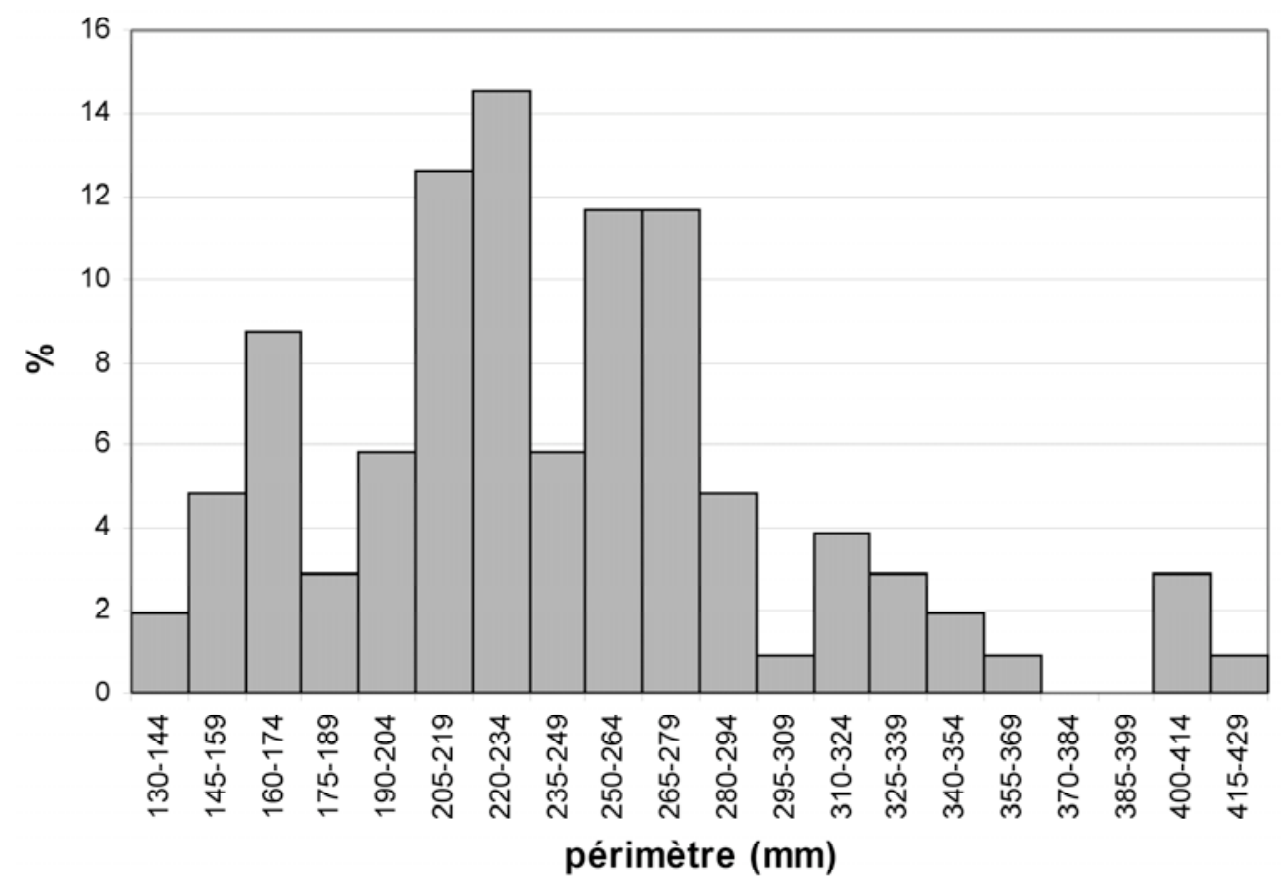

Figure 17 - Répartition des éclats préférentiels entiers d'Hermies en fonction de leur périmètre (talon exclu), tous niveaux confondus.

Figure 17 - Perimeter (without butt) of the unbroken preferential flakes from Hermies, all levels taken into account. 

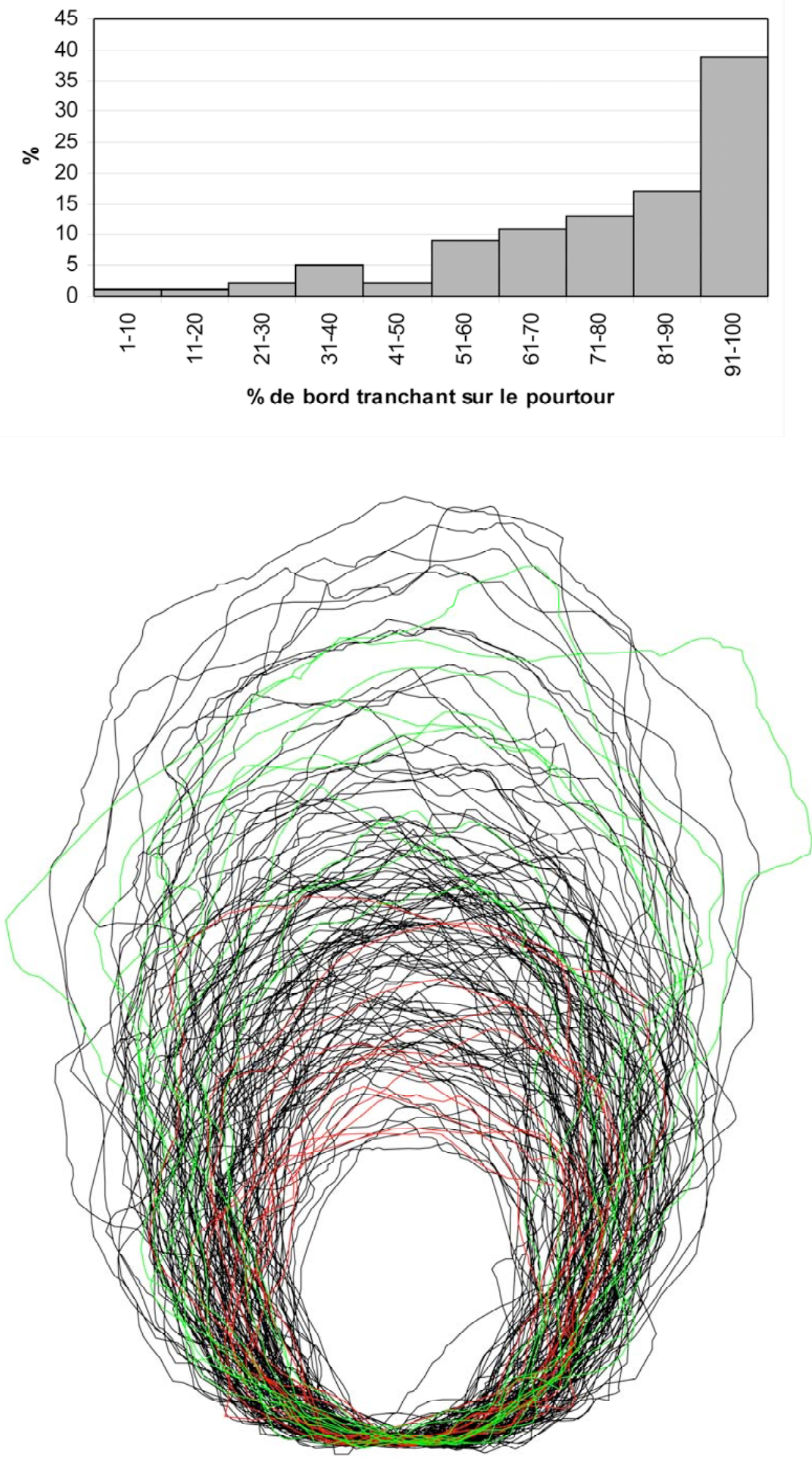

Figure 18 - Part de tranchant utile sur les éclats préférentiels d'Hermies (rapport longueur de tranchant utile/périmètre en abscisses, pourcentage de l'effectif en ordonnées).

Figure 18 - Extent of cutting edge on the preferential flakes from Hermies llength of cutting edge/perimeter on the $x$-axis, percentage on the $y$-axis).

Figure 19 -

Superposition des contours des éclats préférentiels entiers d'Hermies-Le Tio Marché ; éclats rebroussés en rouge, éclats outrepassés en vert (DAO : L. Vallin).

Figure 19 - Outline of unbroken Levallois flakes from Hermies-Le Tio Marché ; hinged flakes in red, plunging flakes in green (CAD : L. Vallin).

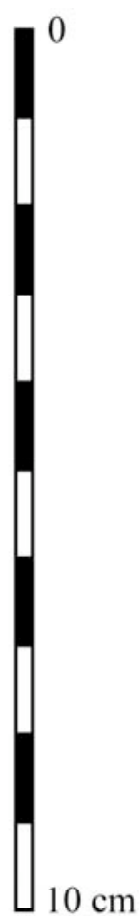


tant un bord droit entièrement abrupt $(n=4)$ est à peu près égal au nombre d'éclats présentant un bord gauche totalement abrupt $(n=6)$, le nombre d'éclats présentant un bord gauche partiellement abrupt $(n=36)$ n'est que légèrement supérieur au nombre d'éclats présentant un bord droit partiellement abrupt $(n=30)$, ce qui coïncide avec les constatations faites plus haut sur les nucléus. De même, les éclats présentant un tranchant plus développé à droite sont sensiblement aussi nombreux que les éclats dont le tranchant gauche est dominant.

La confrontation graphique des contours des éclats Levallois (fig. 19) souligne une certaine homogénéité morphologique d'ensemble, que masquait l'analyse trop fragmentée des bords : si l'on élimine les éclats présentant un accident et qui ne reflètent pas l'intention du tailleur, la quasi-totalité des éclats préférentiels, indépendamment de leur dimension, affectent une forme ovalaire, ce que traduit l'indice d'allongement (tabl. 5) ; dans un certain nombre de cas, les bords convergent vers la partie proximale et non vers la partie distale, offrant parfois une forme spatulée : dans ce dernier cas, les éclats présentent un profil longitudinal légèrement arqué. Les éclats triangulaires ou présentant une pointe dans leur partie distale représentent une catégorie très minoritaire, de même que les éclats allongés. Les formes présentant une dissymétrie latérale sont peu nombreuses.

Par ailleurs, la superposition des contours des éclats Levallois montre une assez large gamme dimensionnelle mais confirme la polarisation autour d'un certain gabarit, surtout si l'on élimine les éclats rebroussés et les éclats outrepassés qui représentent les extrêmes aberrants.

Ces constatations concernent surtout le niveau pléniglaciaire moyen d'Hermies-Tio Marché, qui représente la plus grosse partie de l'effectif étudié. Elles semblent s'appliquer également aux autres niveaux, avec des réserves (pour des raisons taphonomiques) pour les séries provenant des cailloutis.

\section{3 - ADÉQUATION PRODUCTION/UTILISATION : STANDARDISATION AU DEUXIĖME DEGRÉ}

Rechercher une éventuelle normalisation des outils conduit au final à l'identification de critères de choix des objets propres à un (ou plusieurs) usage(s) et, a contrario, de rejet des autres. En d'autres termes, il faut dans un premier temps identifier les pièces utilisées puis dans un deuxième temps rechercher, par l'analyse statistique, si ces objets se distinguent par des caractères particuliers, et secondairement vérifier s'il existe des distinctions selon le type d'utilisation.

\section{1 - Identification des produits utilisés}

Comme sur la plupart des gisements moustériens de plein air du nord de la France, le pourcentage d'éclats retouchés est très faible ; si l'on exclut les pseudo-outils générés par des processus taphonomiques (Caspar et al. 2005), le nombre d'éclats retouchés se restreint à moins d'une dizaine d'individus sur le site du Champ Bruquette et à une trentaine au Tio Marché (racloirs, sur éclat Levallois le plus souvent, et grattoirs). L'écrasante majorité des éclats pré- férentiels ne présente pas de retouches, au sens typologique du terme, ce qui est un trait commun des sites paléolithiques moyens de plein air du nord de la France.

Grâce à l'exceptionnel état de conservation des niveaux d'occupation d'Hermies, on a pu identifier un usage des produits bruts. L'approche tracéologique effectuée par l'un de nous (J.-P. C.) a permis de caractériser deux types de stigmates issus d'utilisations différentes.

L'un, visible seulement à l'échelle microscopique sur 10 pièces, se présente sous la forme d'un micropoli réfléchissant modérément la lumière incidente, contrastant peu avec les surfaces non altérées des silex. Le fil des tranchants est faiblement marqué. Les polis sont bifaciaux, peu envahissants. Les surfaces altérées par l'usage sont modérément régularisées et présentent un aspect gras luisant. Les stries, peu fréquentes, sont très courtes (inférieures à $20 \mu$ ), étroites (inférieures à $2 \mu$ ) et profondes, seulement visibles à un grossissement de 500 fois, localisées sur les deux faces, parallèles ou obliques au tranchant. Ces polis présentent, sur quatre pièces (fig. 20), des analogies avec ceux qui apparaissent expérimentalement lors de l'éviscération, de la découpe de la viande ou, encore, de l'écharnage, du dépiautage et du découpage de la peau fraîche, en cas d'utilisations brèves. Dans plusieurs cas $(n=6)$ cependant, de petits îlots de poli d'os et des stries résultant de l'abrasion due à l'effritement des tranchants actifs accompagnent l'usure. Elle est très semblable, dans cette configuration, aux usures expérimentales obtenues par le dépeçage lors d'activités de boucherie. Deux pièces supplémentaires présentent sur un bord des traces comparables à un poli de bois expérimental. L'état d'avancement de nos travaux sur l'industrie des sites d'Hermies et un retour aux pièces par un examen microscopique plaident pour une interprétation taphonomique de ces traces. En effet, l'aplatissement des dômes du poli en opposition avec la convexité prononcée de ces demiers, caractéristique du poli de bois, pourrait résulter d'une friction sur de la glace plus ou moins chargée en matières abrasives comme nos expérimentations l'ont montré (Caspar et al. 2003). D'ailleurs, l'une de ces deux pièces a été singularisée par l'analyse multivariée et retirée du test de prédiction (cf. infra).

L'autre type de trace, macroscopique, en l'absence de tout stigmate microscopique, se présente, dans neuf cas, sous la forme d'enlèvements plus ou moins envahissants (millimétriques à centimétriques), sur une portion courte $(1 \mathrm{~cm})$ ou étendue (une dizaine de centimètres) de bords simples ou doubles, opposés et/ou adjacents (fig. 21). L'absence d'écrasement sur les fils suggère un travail sur une matière minérale tendre ou encore sur des matériaux organiques durs (os, bois de cervidé, bois durs...). En effet, l'expérimentation montre que des durées d'utilisation même extrêmement courtes (quelques dizaines de secondes) en percussion lancée ou posée avec percuteur sur des matières minérales dures à moyennement dures, auraient entraîné un martelage (ou écrasement) rapide du fil, en plus de l'esquillement (Caspar 1988). L'une des interprétations plausibles, que l'on pourrait conférer à ces objets, serait un usage violent de durée variable sur une matière organique relativement dure, comme le fendage longitudinal des os longs pour récolter la moëlle, par exemple. Pour 

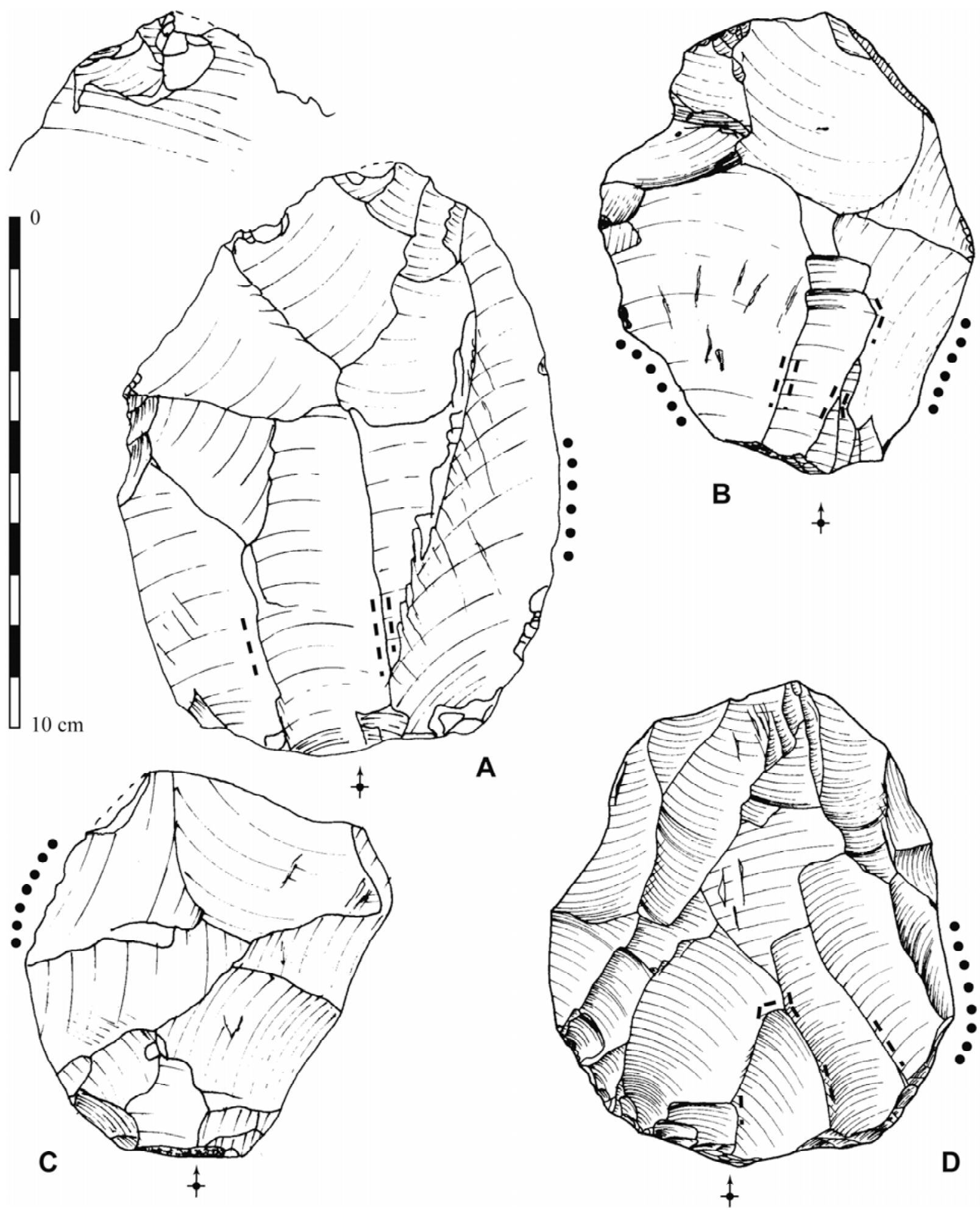

Figure 20 - Eclats utilisés, stigmates d'utilisation microscopique : A-O22/deb1 (poli 50), B-G20/3 (poli 50), C-Q22/26 (poli 30), DD102/3 (poli 50) ; traces possibles d'emmanchement en tireté, position des polis d'usure en pointillé ; A, B et C : Le Tio Marché, $D$ : Le Champ Bruquette.

Figure 20 - Used flakes with micro-use marks : A-O22/deb1 (poli 50), B-G20/3 (poli 50), C-Q22/26 (poli 30), D-D102/3 (poli 50); dash line : possible handling marks, dotted line : location of the use polish; $A, B$ et $C$ : Le Tio Marché, $D$ : Le Champ Bruquette. 

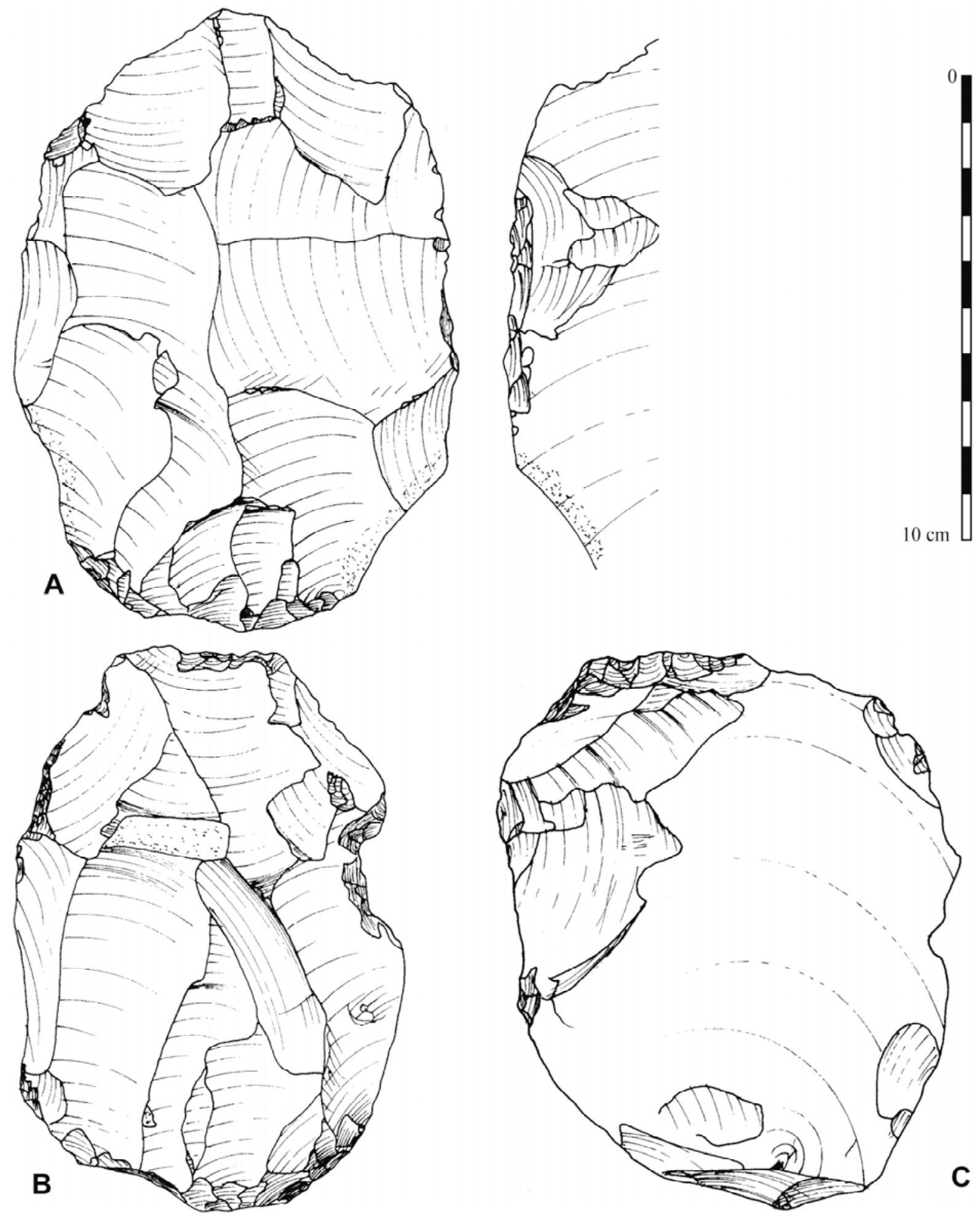

Figure 21 - Eclats utilisés, stigmates d'utilisation macroscopique : A-P23/7 (éclat marqué par un esquillement envahissant inverse et partiel de l'extrémité distale et du bord mésial droit), B-O23/8 (pièce esquillée), C-T21/58 (pièce esquillée présentant également un esquillement du bord distal).

Figure 21 - Used flakes with macro-use marks : A-P23/7, B-O23/8, C-T21/58. 
les pièces présentant des enlèvements sur deux bords opposés $(n=3)$ et proches des pièces esquillées au sens typologique du terme (Tixier 1963), l'on peut imaginer que ces stigmates se sont formés simultanément lors de la même opération technique en percussion posée avec percuteur, avec un bord pénétrant le matériau d'œuvre et l'autre subissant l'action du percuteur (Semenov 1970 p. 149-150 ; Mazière 1984). En ce qui concerne la nature du matériau de ce demier, elle reste indéterminée. L'une des pièces esquillées présente en outre un esquillement alternant du bord distal ; elle pourrait être rattachée à deux autres éclats présentant un esquillement unifacial de l'extrémité distale : celle-ci pourrait coïncider avec la partie active de l'outil, les talons larges et épais opposés recevant la percussion. Un autre éclat est marqué par un esquillement envahissant inverse et partiel de l'extrémité distale et du bord mésial droit. Les trois demières pièces portent un esquillement partiel dans la partie mésiale ou distale d'un bord latéral ; le bord opposé, dans tous les cas de faible angulation, ne montre pas de trace d'utilisation ni de percussion. L'action responsable de ces stigmates est indéterminée.

\section{2 - Approche spatiale}

La confrontation, par les remontages, des nucléus (et des déchets de fabrication) d'une part et des produits finis d'autre part permet de mettre en évidence les déplacements d'objets à l'intérieur du site ou les lacunes, qui peuvent révéler des prélèvements à fin d'usage par les hommes préhistoriques ; un problème de contemporanéité se pose toutefois pour les pièces non remontées. II faut également garder présent à l'esprit que seule une partie de chaque site a pu être fouillée et que le degré de conservation des niveaux archéologiques, s'il est globalement excellent pour les deux occupations principales, est souvent très médiocre pour les autres séries.

En partant du principe (conforté par les remontages et l'étude taphonomique) que les amas reconnus à la fouille correspondent à des amas de taille en place, on a distingué trois types possibles de situation, qui se divisent en cinq sous-types, en fonction de la position des éclats préférentiels par rapport à leur amas de taille d'origine (fig. 22) :

- Catégorie A : l'éclat préférentiel a été retrouvé dans le poste de débitage où il a été produit ; c'est la catégorie la plus aisée à reconnaître ;

- Catégorie B : l'éclat préférentiel a été retrouvé hors du poste de débitage où il a été produit, et dont la position est connue : on peut alors mesurer sa distance de transport ; deux sous-catégories peuvent être distinguées :

B1 : l'éclat préférentiel a été retrouvé dans un autre poste de débitage du même niveau (ce qui n'implique pas une stricte contemporanéité, ni un déplacement important),

B2 : l'éclat préférentiel a été retrouvé à l'écart de tout poste de débitage.

La difficulté consiste dans la définition d'un seuil de distan$\mathrm{ce}$ : nous l'avons mis assez bas (1 mètre) dans la mesure où les concentrations sont souvent proches les unes des autres (au Tio Marché, aucun des éclats préférentiels étudiés n'est éloigné de plus de $4 \mathrm{~m}$ d'un poste de débitage) ; - Catégorie C : l'éclat préférentiel a été retrouvé hors du poste de débitage où il a été produit, et dont la position est inconnue : on ne peut alors mesurer sa distance de transport, qui pourrait d'ailleurs être faible lorsque l'éclat préférentiel se situe près des limites de fouille (au Tio Marché, aucun des éclats préférentiels étudiés ne se trouve à plus de 1,80 $\mathrm{m}$ d'une limite de fouille) ; deux sous-catégories peuvent être distinguées :

C1 : l'éclat préférentiel a été retrouvé dans un autre poste de débitage du même niveau,

$\mathrm{C} 2$ : l'éclat préférentiel a été retrouvé à l'écart de tout poste de débitage.

Pour être tout-à-fait complet, il faudrait créer une catégorie D pour regrouper les éclats " fantômes ", attestés sur certains nucléus préférentiels par le négatif du demier enlèvement mais qui n'ont pas été retrouvés dans les limites de la zone fouillée ; nous n'en avons toutefois pas tenu compte, pour des raisons méthodologiques.

Les catégories $A$ et $B$ représentent la production certainement "domestique ", c'est-à-dire réalisée sur place, la catégorie C pourrait englober les éclats " importés ", avec les produits " domestiques "qui n'ont pu être remontés par défaut d'observation (soit parce que le lieu de production se situait dans une partie non explorée du site, soit parce que les essais de remontage n'ont pas été assez poussés, en particulier dans les zones où les produits des séquences de débitage ont été disséminés) ; en symétrique la catégorie $D$, avec les mêmes réserves, représenterait la production " domestique " exportée du site. On postule que les éclats de la catégorie A n'ont pas été utilisés, puisqu'une utilisation au sein même d'un amas est peu probable, pas plus qu'on puisse imaginer que le produit ait été replacé, après usage, dans l'amas. En revanche, la probabilité d'usage (ou d'intention d'usage) des éclats des catégories B2 et C2 (et $D$ ) est, en théorie, d'autant plus élevée que la distance du lieu de production connu (B2) ou possible (C2, D) augmente. L'interprétation des catégories B1 et C1 est plus problématique ; il existe, sur les deux sites, de rares cas d'" échanges " entre postes de débitage, mais ils concernent plutôt des nucléus. La distribution spatiale de ces derniers semble d'ailleurs obéir aussi à des règles spécifiques qu'il faudrait peut-être croiser avec les mouvements des éclats préférentiels.

Ce schéma d'interprétation dynamique est toutefois faussé par des processus post-dépositionnels d'origine géologique (cryoturbation, solifluxion, ruissellement, etc.), animale ou humaine (piétinement entre autres) : dans certains secteurs du Tio Marché, c'est en effet l'ensemble des produits d'une séquence de réduction qui peut être disséminé sur plusieurs mètres carrés. En ce sens, l'approche spatiale ne peut fournir qu'une tendance globale et ne peut permettre une diagnose d'utilisation individuelle, à la différence de la tracéologie.

Il convient de préciser que l'approche spatiale a été menée indépendamment, l'analyse fonctionnelle étant faite en aveugle. Seuls les résultats de cette demière ont été pris en compte dans la base de données soumise à l'étude statistique, la confrontation avec l'analyse spatiale n'intervenant qu'ensuite (tabl. 6).

Dans l'état actuel des remontages, deux constatations peuvent être faites : un seul éclat de la catégorie A pré- 


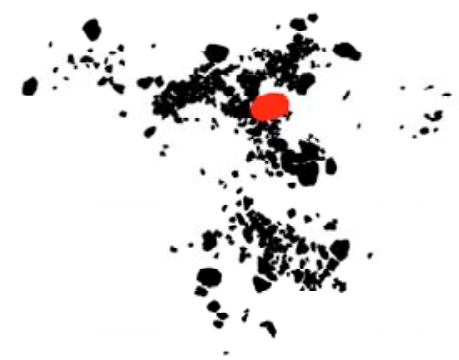
A : l'éclat préférentiel a été retrouvé dans le poste de débitage où il a été produit

B : l'éclat préférentiel a été retrouvé hors du poste de débitage où il a été produit, et dont la position est connue ; deux sous-catégories peuvent être distinguées :

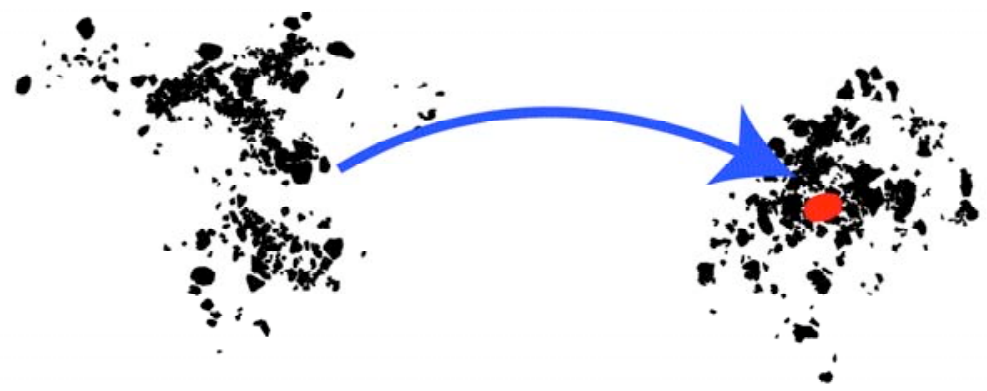

B1 : l'éclat préférentiel a été retrouvé dans un autre amas

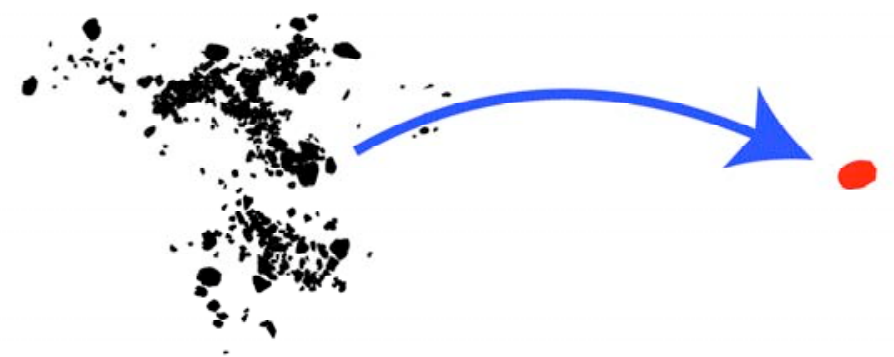

B2 : l'éclat préférentiel a été retrouvé à l'écart de tout poste de débitage

C : l'éclat préférentiel a été retrouvé hors du poste de débitage où il a été produit, et dont la position est inconnue ; deux sous-catégories peuvent être distinguées :

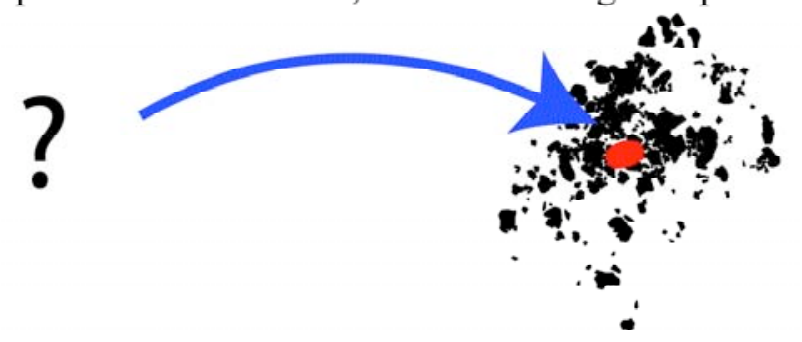

C1 : l'éclat préférentiel a été retrouvé dans un autre amas

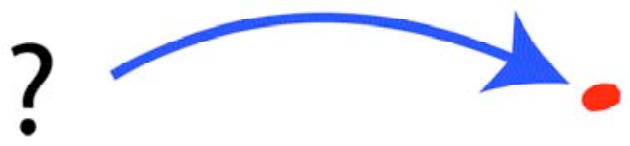

C2 : l'éclat préférentiel a été retrouvé à l'écart de tout poste de débitage

Figure 22 - Situation des éclats préférentiels par rapport à la chaine opératoire : catégories distinguées (DAO : B. Masson).

Figure 22 - Preferential flakes position in the chaine opératoire : different groups (CAD : B. Masson). 


\begin{tabular}{|l|c|c|c|c|}
\hline & $\begin{array}{l}\text { Total éclats } \\
\text { Tio Marché }\end{array}$ & $\begin{array}{l}\text { Eclats utilisés } \\
\text { Tio Marché }\end{array}$ & $\begin{array}{l}\text { Total éclats } \\
\text { Champ Bruquette }\end{array}$ & $\begin{array}{l}\text { Eclats utilisés } \\
\text { Champ Bruquette }\end{array}$ \\
\hline A & 12 & 1 & 13 & 0 \\
\hline B1 & - & - & - & 0 \\
\hline B1 ou B2 & 4 & - & - & 0 \\
\hline B2 & 3 & 2 & 1 & 0 \\
\hline C1 & 2 & 1 & 3 & 3 \\
\hline C2 & 16 & 9 & 2 & 0 \\
\hline A ou B & 11 & - & - & 0 \\
\hline A ou C & 2 & 1 & 6 & 0 \\
\hline B ou C & 36 & 4 & 2 & 3 \\
\hline $\begin{array}{l}\text { Absence de sol } \\
\text { archéologique }\end{array}$ & 13 & 1 & 28 & 0 \\
\hline Total & 99 & 19 & & \\
\hline
\end{tabular}

Tableau 6 - Classification des éclats préférentiels d'Hermies pris en compte dans cette étude (tous niveaux confondus) en fonction de leur position spatiale par rapport à la chaîne opératoire.

Table 6 - Classification of the preferential flakes taken into account in this paper (all levels) from the point of view of the spatial location regard to the chaine opératoire.

sente des stigmates d'utilisation (au Tio Marché), toutefois il se trouve également très proche d'une concentration d'éclats préférentiels "importés " qui portent tous des stigmates d'utilisation ; la catégorie C2 comprend la majorité des éclats préférentiels visiblement utilisés, voire la quasitotalité si l'on ajoute les éclats sur lesquels la prudence nous a retenus. En revanche, un fort pourcentage des éclats " déplacés " ne présentent pas de stigmates visibles d'utilisation, ce qui n'exclut pas une utilisation brève ou de faible intensité. D'après leur position dans la chaîne opératoire et en tenant compte de paramètres méthodologiques et taphonomiques, le nombre d'éclats préférentiels extraits de leur lieu de débitage se monterait à 67 , parmi lesquels on trouve 19 pièces effectivement utilisées, d'après l'analyse tracéologique.

\section{3 - Reconnaissance statistique de critères de sélec- tion des produits utilisés}

L'identification d'un échantillon —à défaut de la totalitéde produits utilisés permet de rechercher les exigences des utilisateurs, au moyen de l'analyse statistique; celle-ci a été effectuée par l'un de nous (E. D.).

\subsection{1 - Analyses univariées}

L'analyse de la variance à un critère de classification (ANOVA I) a été utilisée pour comparer les moyennes des 3 groupes : NU (non utilisés, $n=106)$, MiU (microtraces d'utilisation, $n=12$ ) et $\mathrm{MaU}$ (macrotraces d'utilisation, $n=9$ ) sur l'ensemble des variables mesurées. Cette technique d'analyse permet de comparer les moyennes de plus de deux

\begin{tabular}{|l|c|c|c|}
\hline & NU & \multicolumn{1}{l|}{ MiU } & $\mathrm{MaU}$ \\
\hline Nombre & 106 & 12 & 9 \\
\hline PROFIL LONGITUDINAL RECTILIGNE & $0,4 \mathrm{a}$ & $1,0 \mathrm{~b}$ & $0,9 \mathrm{~b}$ \\
\hline BORD MODIFIÉ DISTAL & $0,2 \mathrm{a}$ & $0,5 \mathrm{~b}$ & $0,7 \mathrm{~b}$ \\
\hline BORD MODIFIÉ DROIT DISTAL & $0,1 \mathrm{a}$ & $0,1 \mathrm{a}$ & $0,7 \mathrm{~b}$ \\
\hline BORD UTILISÉ GAUCHE PROXIMAL & & $0,3 \mathrm{a}$ & $0,1 \mathrm{~b}$ \\
\hline BORD UTILISÉ GAUCHE DISTAL & & $0,3 \mathrm{a}$ & $0,3 \mathrm{a}$ \\
\hline BORD UTILISÉ DISTAL & & $0,1 \mathrm{a}$ & $0,7 \mathrm{~b}$ \\
\hline BORD UTILISÉ DROIT DISTAL & & $0,7 \mathrm{a}$ & $0,7 \mathrm{a}$ \\
\hline BORD UTILISÉ DROIT PROXIMAL & & $0,5 \mathrm{a}$ & $0,3 \mathrm{a}$ \\
\hline ESQUILLES PROXIMAL GAUCHE & $0,1 \mathrm{a}$ & $0,4 \mathrm{a}$ & $0,8 \mathrm{~b}$ \\
\hline ESQUILLES PROXIMAL DROIT & $0,1 \mathrm{a}$ & $0,4 \mathrm{a}$ & $0,7 \mathrm{~b}$ \\
\hline
\end{tabular}

Tableau 7 - Moyenne d'une sélection de variables dans les 3 groupes (NU = non utilisés, MiU = stigmates d'utilisation microscopique, MaU=stigmates d'utilisation macroscopiques). Les valeurs significativement plus élevées $(P<0,05)$ d'un groupe à l'autre sont annotées par une lettre différente $(a, b)$.

Table 7 - Average of some variables in the 3 groups (NU = non used, MiU = microscopic use-marks, MaU = macroscopic use-marks). Significant highest values $(P<0.05)$ are distinguished by different letters $(a, b)$. 


\begin{tabular}{|l|c|c|c|c|c|}
\hline site & TM & CB & CB/TM & TM & CB \\
\hline Nombre & 99 & 28 & & 99 & 28 \\
\hline & \multicolumn{2}{|c|}{ Moyenne } & $\%$ & \multicolumn{2}{|c|}{ Ecart-type } \\
\hline FISSURATION & 0,15 & 0,32 & 212 & 0,36 & 0,48 \\
\hline ALTÉRATION & 0,05 & 0,18 & 354 & 0,22 & 0,39 \\
\hline PROFIL ARQUÉ & 0,11 & 0,29 & 257 & 0,32 & 0,46 \\
\hline REBROUSSEMENT DISTAL & 0,18 & 0,36 & 196 & 0,39 & 0,49 \\
\hline BORD SINUEUX GAUCHE PROXIMAL & 0,08 & 0,21 & 265 & 0,27 & 0,42 \\
\hline BORD CONVEXE GAUCHE DISTAL & 0,29 & 0,11 & 37 & 0,46 & 0,31 \\
\hline BORD SINUEUX GAUCHE DISTAL & 0,09 & 0,32 & 354 & 0,29 & 0,48 \\
\hline
\end{tabular}

Tableau 8 - Moyennes, rapport entre les moyennes et écart-types pour les variables présentant des différences significatives d'un site à l'autre (TM = Tio Marché, CB = Champ Bruquette).

Table 8 - Averages, relation between the averages and standard deviation of variables showing significative differences between the two sites (HTM = Hermies-Le Tio Marché, HCB = Hermies-Le Champ Bruquette).

échantillons (le test de $\mathrm{t}$ de Student, largement pratiqué, peut être considéré comme un cas particulier de l'ANOVA à un critère de classification lorsqu'il n'y a que deux groupes expérimentaux en présence). L'ANOVA I a été suivie de comparaisons pairées par la méthode de Scheffé. Le seuil de signification de référence est le seuil $5 \%$.

II apparait qu'un très grand nombre de variables dont nous ne reprendrons pas la liste ici, mais qui sont essentiellement liées à la taille de la pièce, sont de moyenne significativement plus élevées pour les éclats présentant des stigmates macroscopiques d'utilisation, ce qui corrobore l'idée que les individus de ce groupe sont généralement de grande taille, bien que la représentation des variables liées à la taille dans des histogramme univariés montre un recouvrement des groupes : cette plus grande taille n'est pas systématique, mais juste une tendance moyenne.

Les variables présentant une différence significative et permettant une interprétation plus fine sont reprises dans le tableau 7.

La variable PROFIL LONGITUDINAL RECTILIGNE montre que l'absence de cambrure de la pièce est un élément très discriminant de son utilisation, la quasi-totalité des éclats préférentiels utilisés ayant un tel profil. La modification du bord distal apparaît liée à l'utilisation, quel qu'en soit le type, tandis que la modification du bord droit distal apparaît liée à la présence de stigmates macroscopiques d'utilisation.

Les bords utilisés à droite (BORD UTILISE DROIT DISTAL et BORD UTILISE DROIT PROXIMAL) apparaissent plus fréquents que leurs symétriques gauches (BORD UTILISE GAUCHE PROXIMAL et BORD UTILISE GAUCHE DIS$T A L)$, laissant supposer une latéralité plus marquée à dro ite. La variable BORD UTILISE DISTAL est caractéristique du groupe MaU (macrotraces d'utilisation). Une légère surutilisation du bord gauche proximal apparaît dans le gro upe MiU (microtraces d'utilisation).

Quant aux esquilles aux points encadrant le talon (ESQUILLES EN PROXIMAL GAUCHE et ESQUILLES EN
PROXIMAL DROIT), qui peuvent être un rognage facilitant la préhension, elles sont quasi-absentes des pièces du groupe NU (non utilisées) et significativement plus élevées dans le groupe MaU (macrotraces d'utilisation).

Nous avons ensuite tenté de mettre en évidence une éventuelle différence de standard de fabrication entre les sites du Champ Bruquette et du Tio Marché. Le site du Champ Bruquette ne présentant pas de pièces cataloguées MaU, il serait trivial de mettre en évidence que les variables permettant d'identifier le groupe MaU identifient également le Tio Marché. Nous avons donc réalisé une comparaison des moyennes entre le Champ Bruquette et le Tio Marché pour les variables ne présentant pas de différences entre MaU et le reste (reprises dans le tableau 8).

La première constatation est que la qualité de la matière première (traduite, entre autres, par la variable FISSURATION) est moindre au Champ Bruquette, caractéristique qui pourrait être en partie corrélable à des défauts de fabrication plus élevés sur ce site (PROFIL ARQUE, REBROUSSEMENT DISTAL), à des bords plus sinueux (BORD SINUEUX GAUCHE PROXIMAL, BORD SINUEUX GAUCHE DISTAL) et à moins de bords convexes propices à l'utilisation (BORD CONVEXE GAUCHE DISTAL). L'altération y est plus importante. Aucune variable permettant de conclure à une différence de technicité ou de standard de fabrication n'a été mise en évidence par cette analyse.

\subsection{2 - Analyses multivariées}

L'analyse en composantes principales (ACP) est l'une des techniques exploratoires les plus utilisées en analyse multivariée. Purement descriptive, elle n'implique aucun modèle de normalité et ne propose pas de seuil statistique. Elle consiste en une décomposition d'une matrice de corrélation entre les variables originales en vecteurs propres, qui représentent des directions orthogonales dans l'espace (et donc statistiquement indépendantes) et en valeurs propres, qui expriment une variance (mélange d'information et de bruit de fond). Le but est de représenter dans un 
petit nombre de dimensions (idéalement un plan) un maximum de la variance liée à l'information et un minimum de la variance liée au bruit de fond. Son interprétation, intuitive, repose sur trois informations complémentaires :

(a) La proportion de variance exprimée dans le plan, qui reflète la qualité de la projection des points sur le plan. Plus la proportion de variance est élevée, plus la proximité des points observée sera proche de celle de leur représentation de l'hyperespace original (qui a autant de dimensions qu'il y a de variables dans les données expérimentales).

(b) la répartition des objets (ici les pièces) dans le plan. Des groupes de points proches représentent des groupes d'objets aux caractéristiques plus ou moins homogènes.

(c) la corrélation entre les variables originales et les axes du plan. Les variables fortement corrélées à un axe permettent de lui donner une " étiquette " permettant d'interpréter cette construction mathématique abstraite dans les termes du contexte expérimental.
Toutes les mesures radiaires prises de $10^{\circ}$ en $10^{\circ}$ ont été soumises à une analyse en composantes principales (ACP) destinée à différencier la taille et la forme des 127 pièces (Jolicoeur et Mosimann 1960). Lorsqu'un ensemble d'objets de taille variable se répartit en plusieurs groupes de forme distincte, le premier axe de l'ACP exprime la variation liée à la taille et le second celle liée aux différences de forme. Cette analyse a, au contraire, mis en évidence un continuum entre les différentes pièces, montrant qu'il s'agit de variants d'une même forme de base, sans différence marquée entre différents gabarits, qui auraient pu correspondre au type d'utilisation (ou à la non utilisation) des pièces. Cette observation recoupe celle qui peut être obtenue en superposant graphiquement les profils des différentes pièces (fig. 19 et 23).

Après avoir caractérisé les pièces, nous avons tenté de détecter celles qui auraient toutes les caractéristiques des

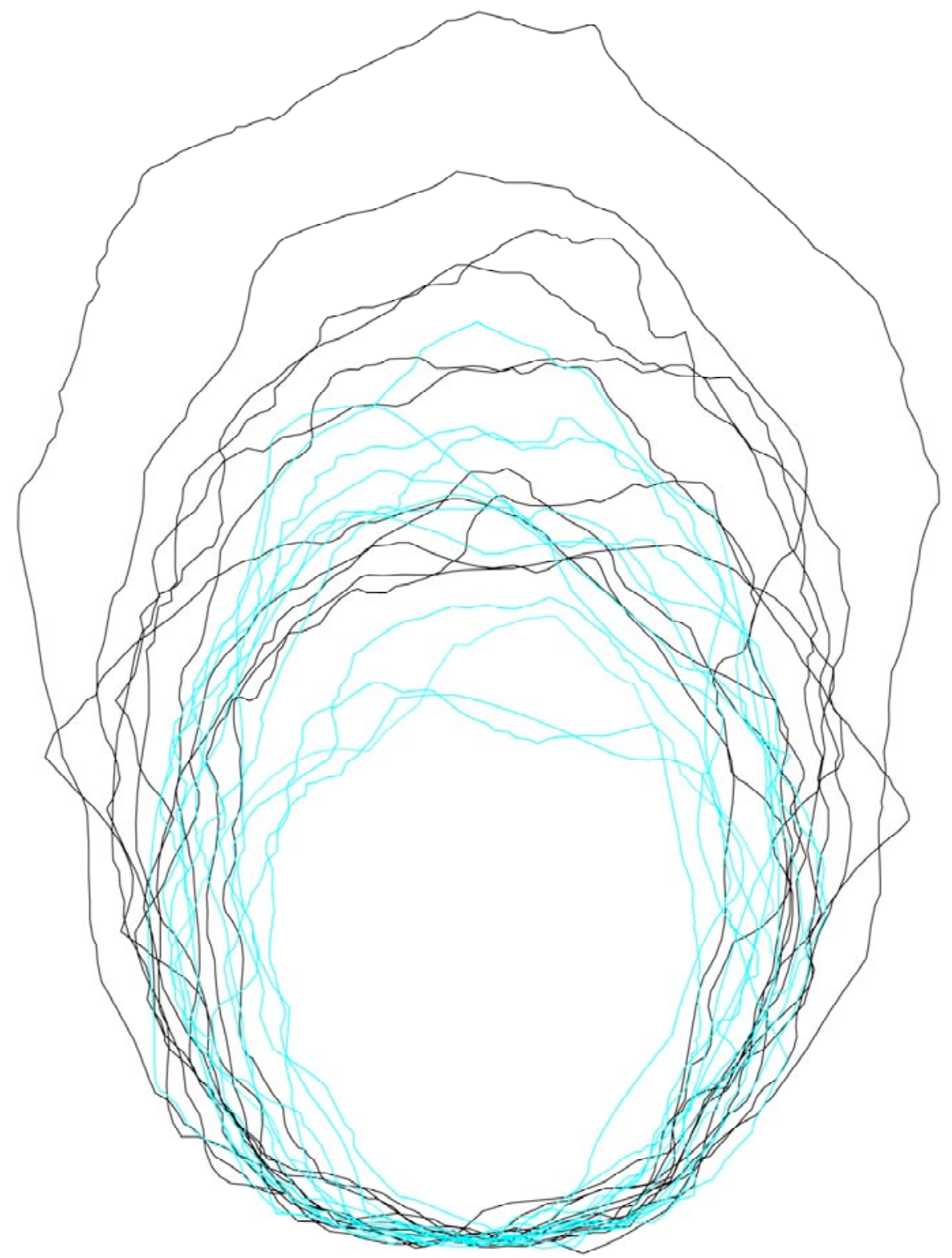

Figure 23 - Superposition des contours des éclats Levallois utilisés, avec stigmates d'utilisation microscopique en bleu, avec stigmates d'utilisation macroscopique en noir (DAO : L. Vallin).

Figure 23 - Superposing of used Levallois flakes outlines ; blue line: used flakes with micro-use marks, black line : used flakes with macro-use marks (CAD : L. Vallin).

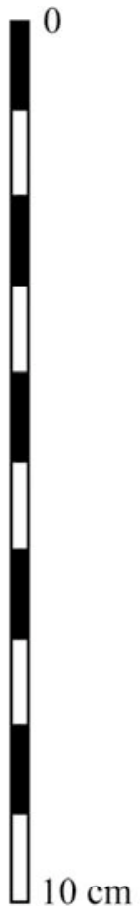


pièces utilisables mais qui n'auraient pas été utilisées (tout au moins de façon perceptible). Nous avons eu recours à l'analyse discriminante pas-à-pas. Cette technique est un autre type d'analyse multivariée, que nous avons utilisé ici dans sa version descriptive, les conditions d'inférence n'étant pas réunies. Son but est de rechercher la combinaison de variables qui permet la meilleure discrimination entre des groupes d'objets préalablement définis, qui correspondent ici aux groupes NU $(n=106)$, MiU $(n=12)$ et $\mathrm{MaU}(\mathrm{n}=9)$, afin de définir la pertinence des groupes, leur degré de recouvrement et les variables essentielles pour les classer. L'analyse a été réalisée sur toutes les variables présentant une variance non nulle dans chacun des 3 groupes considérés.

Une première analyse fait ressortir deux pièces relativement mal placées dans leur groupe. La pièce P6/37, identifiée MiU en raison de la présence d'un micro-poli sur le bord distal, est plus proche des MaU suivant les variables BORD UTILISE DISTAL, BORD MODIFIE DROIT DISTAL, ANGLE BORD GAUCHE PROXIMAL, LARGEUR, POIDS, NOMBRE ENLEVEMENTS ; en réalité, le poli, réparti en ilôts épars, peu développé, proche de celui qui résulte du travail du bois, pourrait être d'origine taphonomique et les enlèvements envahissants sur les deux bords latéraux (inverses à gauche et alternants à droite), d'abord considérés comme le résultat d'un façonnage, pourraient effectivement résulter d'une utilisation comme fendoir d'os longs (cf. supra). La pièce P22/24, identifiée MaU mais plus petite (longueur : $96 \mathrm{~mm}$ )

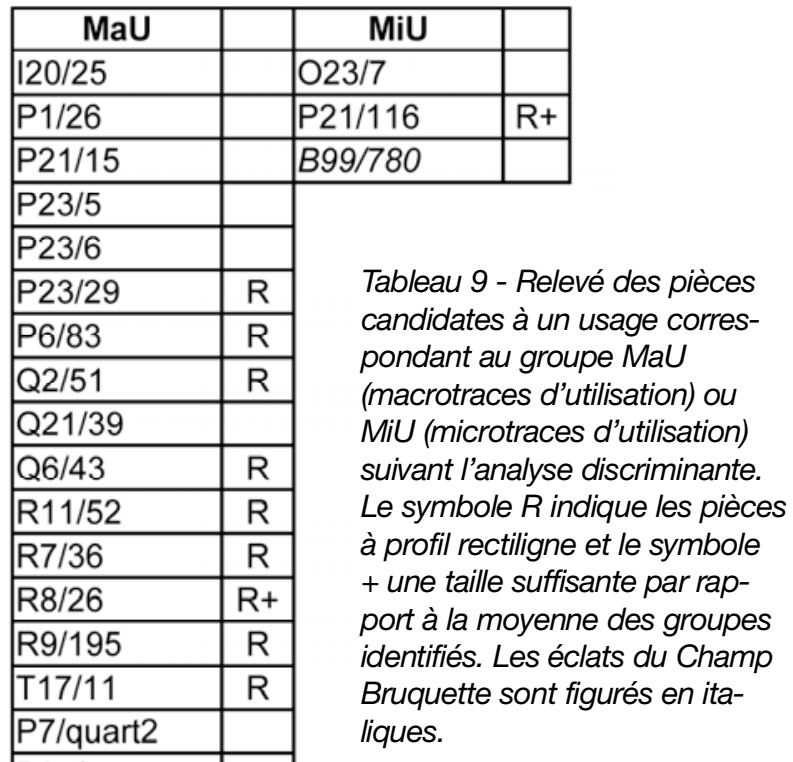

Table 9 - List of possible integration of flakes by discriminant analysis in the groups $\mathrm{MaU}$ (macroscopic use-marks) and MiU (microscopic use-marks). The letter $R$ indicates the flakes with straight profile and the symbol + indicates a size in respect of the group. The Champ Bruquette flakes are italicized. que la moyenne des MaU, est plus proche de MiU suivant les variables ANGLE BORD GAUCHE PROXIMAL, BORD MODIFIE DISTAL, BORD UTILISE DISTAL, BORD MODIFIE DROIT DISTAL, LONGUEUR TRANCHANT BORD DROIT DISTAL et PERIMETRE SANS TALON : les traces d'utilisation macroscopiques, localisées sur le bord distal gauche, sont d'ailleurs beaucoup plus discrètes que sur les autres représentants du groupe.

Etant donné que les groupes MiU et MaU sont petits, un éventuel " intrus " aura beaucoup d'influence subséquente ; plutôt que de modifier a posteriori leur affectation, ils ont été supprimés de la seconde analyse, qui a proposé des groupes relativement homogènes, enrichis de 25 candidats MaU et trois candidats MiU sélectionnés parmi les NU (tabl. 9).

Parmi les 28 pièces proposées, seules 13 présentent un profil rectiligne : 1 pièce candidate à un usage correspondant au groupe MiU et 12 au groupe MaU. Nous n'avons ré-examiné que ces 13 objets.

La pièce candidate à un usage correspondant au groupe MiU est P21/116 (fig. 13), mais elle présente un bord gauche proximal cortical et, sur le reste du pourtour, des festons défavorables à l'utilisation en boucherie ou sur une matière animale tendre ; ses dimensions, cependant, la rapprochent du groupe MaU (longueur : $122 \mathrm{~mm}$ ). Quelques retouches écailleuses, dans une zone partiellement saccharoïde, affectent d'ailleurs l'angle distal du bord gauche, correspondant soit à des retouches spontanées, soit à une action taphonomique ou encore à une brève utilisation en percussion lancée ou posée avec percuteur ; des esquillements sont présents sur les portions de bord gauche et droit adjacentes au talon.

Les pièces candidates à un usage correspondant au groupe MaU sont plus nombreuses ; pour le Tio Marché, il s'agit des objets suivants :

$\mathrm{P} 23 / 29$ est une pièce régulière mais cassée au niveau du talon lors du débitage et elle présente un bord droit débordant en proximal ; sa taille est en deçà de celle du groupe MaU (longueur : $72 \mathrm{~mm}$ ) ;

P6/83 (fig. 15) est vraisemblablement trop petit (longueur : $58 \mathrm{~mm}$ ) pour une utilisation correspondant au groupe MaU, l'identification comme éclat préférentiel est d'ailleurs incertaine ;

Q2/51 présente une taille limite par rapport au groupe MaU (longueur : $98 \mathrm{~mm}$ ) ; on observe cependant quelques enlèvements inverses sur le bord distal qui ont été jugés non diagnostiques d'un usage par la présence d'une cassure clairement post-dépositionnelle sur la moitié distale du bord gauche ; en outre, cette pièce reposait sur un cailloutis ;

Q6/43 présente un bord gauche irrégulier ; sa taille est légèrement en deçà de celle du groupe correspondant (Iongueur : $86 \mathrm{~mm}$ )

$\mathrm{R} 11 / 52$ présente une dimension adéquate (longueur : 105 $\mathrm{mm}$ ), mais c'est une pièce de topographie irrégulière, torse, ce qui pouvait présenter un obstacle à son utilisation en cinématique longitudinale posée ;

$\mathrm{R} 7 / 36$ est une pièce régulière, mais apparemment trop petite pour ce type d'utilisation (longueur $67 \mathrm{~mm}$ ) ; on notera la présence de retouches adjacentes au talon sur le bord gauche; R8/26 possède un bord cortical opposé à un bord qui présente des retouches directes irrégulières, de délinéation den- 
ticulée et des cassures en croissant d'origine taphonomique : on ne peut pas exclure une utilisation brève. Ses dimensions coïncident avec celles de ce groupe (longueur : $132 \mathrm{~mm}$ ); R9/195 est vraisemblablement trop petit (longueur : 63 $\mathrm{mm}$ ), il présente un défaut du bord distal, des cassures en croissant post-dépositionnelles sur presque toute la périphérie et des retouches en encoche, d'origine indéterminée, sur le bord gauche proximal ;

T17/11 (fig. 10, C) est trop petit (longueur : $90 \mathrm{~mm}$ ) et surtout trop mince (épaisseur : $13 \mathrm{~mm}$ ) ;

O5/quart 1 présente une taille limite (longueur : $99 \mathrm{~mm}$ ) et un défaut du bord distal ; en outre, le bord gauche est cortical et opposé à un bord droit très irrégulier à cause d'un défaut dans le silex ; il est à noter que cet éclat n'appartient probablement pas au même niveau chronologique que les précédents.

Les deux éclats préférentiels du Champ Bruquette candidats à un usage correspondant au groupe MaU ont été examinés, même si ce type d'usage est inconnu dans la partie explorée du gisement :

AA98/b19 présente des dimensions vraisemblablement trop faibles (longueur : $75 \mathrm{~mm}$ ) ;

AF70 serait utilisable, ses dimensions le rapprochent de ce groupe (longueur : $104 \mathrm{~mm}$ ) ; on notera la présence de retouches ponctuelles sur le bord droit adjacent au talon. Cet examen limite à trois ou quatre les pièces apparemment "récupérables " dans le groupe NU ; elles se placent dans les catégories $\mathrm{C} 1$ et $\mathrm{C} 2$. Cependant, une des pièces $(R 7 / 36)$ prédites par l'analyse multivariée comme appartenant au groupe MaU présente des caractéristiques compatibles avec le groupe MiU ; toutes les autres pièces présentent des bords incompatibles avec un usage en percussion posée longitudinale.

Toutes les analyses ont été réalisées à partir de feuilles de calcul Excel par le logiciel Statistica de StatSoft.

\section{4 - DISCUSSION}

Sur les deux sites étudiés, qui appartiennent au même micro-territoire, le premier et le plus fort critère de choix d'un outil est d'ordre technologique : c'est la sélection de l'éclat préférentiel puisque, si de rares éclats de préparation ont été retouchés, l'examen microscopique d'un échantillonnage de sous-produits, incluant ces éclats retouchés, montre qu'aucun n'a été utilisé de façon manifeste (nous faisons abstraction des pierres ou des débris de débitage présentant des stigmates de percussion, fréquents au Tio Marché). Même les grands éclats à dos cortical ou brut de débitage ne semblent pas utilisés, malgré leur bonne adéquation à une utilisation en percussion posée avec percuteur, ou comme couteau.

La variabilité des produits finis, représentés par les éclats préférentiels, est relative : elle affecte plus les dimensions que la forme générale de l'objet dont le gabarit est assez calibré (éclat ovalaire exclusivement, quel que soit le niveau archéologique) ; elle traduit plus les niveaux de compétence des tailleurs (ou la qualité de la matière première) que la diversité réelle des intentions. En faisant abstraction des aléas, on peut considérer que les tailleurs d'Hermies -au moins ceux du Tio Marché- voulaient pro- duire, selon une méthode standardisée, une gamme dimensionnelle assez large d'un seul et même modèle. Les pièces utilisées présentent une variabilité encore moindre, qui peut d'ailleurs tenir artificiellement du nombre de variables prises en compte, dont certaines n'ont pas d'implication fonctionnelle. Un critère de rejet absolu ressort de l'analyse statistique, l'existence d'un profil non rectiligne (une seule pièce légèrement rebroussée en partie distale a été utilisée : P22/8 et il n'y a pas de pièce arquée ni de pièce outrepassée qui ait été utilisée). La présence d'un défaut du bord distal est un critère de rejet plus ou moins redondant avec le précédent : seuls quatre éclats utilisés présentent ce défaut, encore celui-ci est-il minime (léger rebroussement ou ondulation : Q22/26, P22/8, G20/3 ou léger outrepassage : R22/25) ; c'est probablement une question d'ergonomie, une discontinuité du bord s'opposant à une utilisation correcte. On peut faire un parallèle avec le site de Bettencourt-Saint-Ouen (Somme) où l'analyse fonctionnelle a mis en évidence la recherche de pointes Levallois non arquées pour une utilisation (boucherie) équivalente à celles des éclats d'Hermies (Locht 2002) ; la différence tient à la dimension des outils utilisés, nettement moindre sur ce dernier site.

Par ailleurs, les éclats préférentiels utlisés d'Hermies possèdent en commun une certaine symétrie d'ensemble et une continuité du fil qui échappent à la quantification, d'autant plus que l'esquillement des bords par utilisation, pour les pièces utilisées en percussion posée avec percuteur, gêne la lecture de la délinéation originelle des bords. La présence d'une retouche sur les portions de bords adjacentes au talon est un critère diagnostique et non un critère de choix, puisque ce ne sont pas des caractéristiques intrinsèques, produites au débitage (fig. 24) ; il existe cependant une forte corrélation entre leur présence et l'utilisation de l'éclat; les rares éclats préférentiels non utilisés qui portent ces retouches particulières présentent une symétrie et une régularité qui les signalent à l'attention. On peut les interpréter soit comme des marques de tailleur (Ploux 1989) soit, plus vraisemblablement, comme des aménagements liés à l'emmanchement, ou au confort d'utilisation à main nue : leur position est symétriquement opposée au tranchant utilisé pour ne pas entailler la paume. En ce qui concerne l'emmanchement, l'épaisseur du bulbe des éclats préférentiels, débités au percuteur de pierre dure (cf. supra), pourrait être un obstacle ; certaines pièces utilisées ou "utilisables" présentent des traces morphologiquement comparables à un poli de bois sur les arêtes de la partie proximale (R22/25 et G20/3, D102/3, F103/71, AF70, O21/déb1) mais la convergence possible avec des stigmates d'origine taphonomique (Caspar et al. 2003) incite à la prudence.

Pour résumer, les tailleurs d'Hermies effectuaient un premier choix au niveau de l'acquisition de la matière première en sélectionnant, dans une source de matière première unique, des blocs de dimension et de morphologie adaptées au seul schéma opératoire mis en oeuvre dans leurs ateliers ; ils s'attachaient à produire, selon une méthode prédéterminée contraignante et avec plus ou moins de succès, un seul modèle de produit fini dont la variabilité se situe essentiellement dans les dimensions. Dans cette gamme de production, à laquelle il faudrait peut-être ajou- 

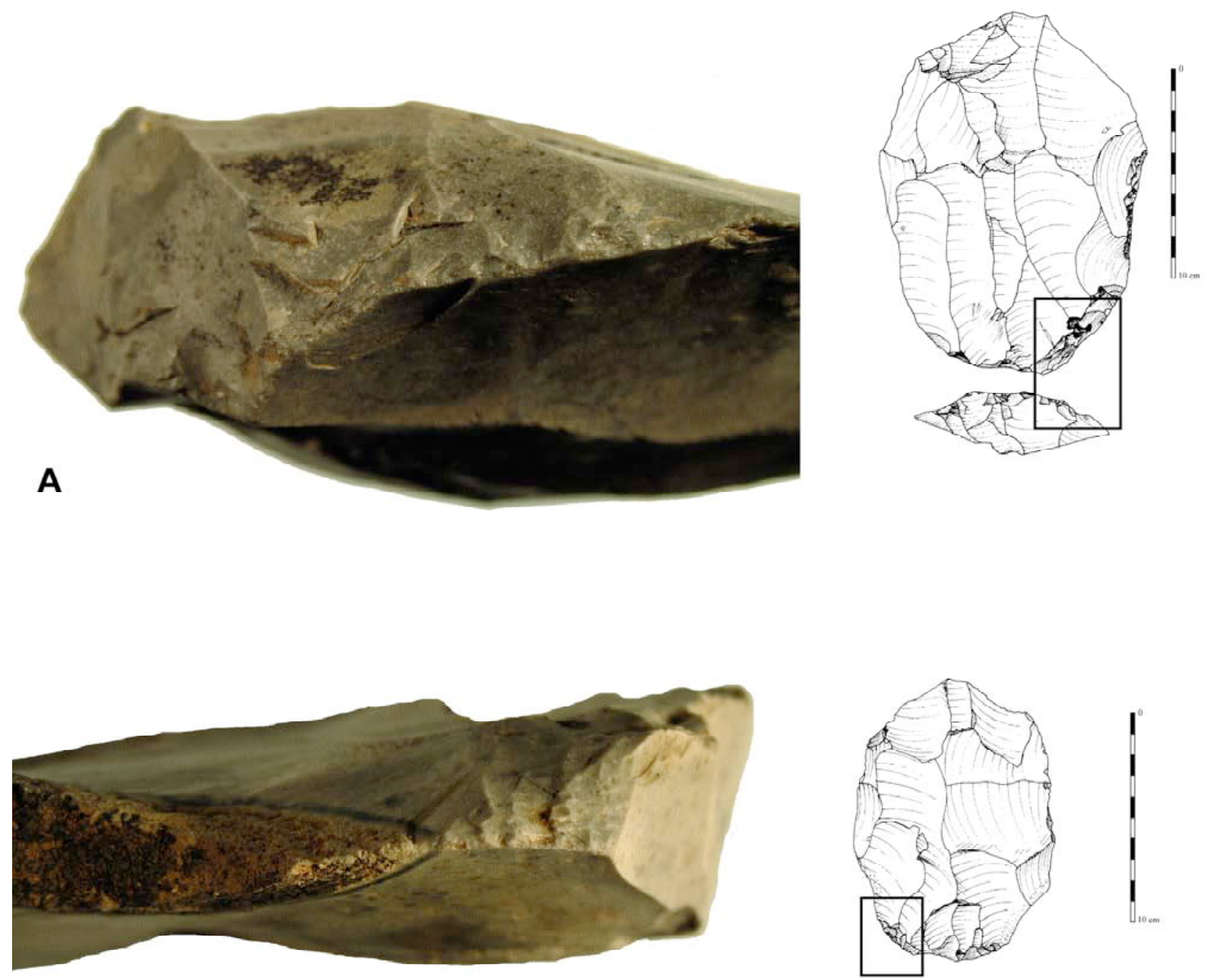

B

Figure 24 : Retouches adjacentes au talon sur des éclats préférentiels utilisés d'Hermies-Le Tio Marché ; A-R23/1, B-P23/7.

Figure 24 - Small retouches near the butt of used preferential flakes from Hermies-Le Tio Marché ; A-R23/1, B-P23/7.

ter une production " exogène ", mais conduite selon le même schéma, étaient sélectionnés pour usage les seuls éclats présentant un profil rectiligne et régulier ; les éclats les plus gros étaient utilisés de préférence pour une percussion posée avec percuteur, les éclats les plus petits pour une percussion posée longitudinale, ces deux actions étant probablement liées dans le cadre d'une activité de boucherie (au sens large). Le recouvrement entre les deux types d'outil et la difficulté à discriminer statistiquement les deux groupes montrent en effet que ces deux gabarits n'étaient probablement pas bien définis lors du débitage (fig. 23).

Se pose alors la question du rapport numérique entre production et utilisation : le pourcentage de pièces utilisées (et utilisables) est très faible, peut-être à nuancer en raison du phénomène d'échantillonnage lié à l'étendue de la fouille. La question se pose aussi de la destination des éclats préférentiels à dos, produits manifestement de façon volon- taire et en nombre notable, selon un procédé d'ailleurs assez standardisé, mais qui ne semblent pas trouver leur place dans une catégorie fonctionnelle quelconque.

La production domestique représente au moins un quart à la moitié des éclats préférentiels abandonnés sur chaque site (cf. supra et tabl. 6) (proportion probablement sousévaluée, d'autant que les éclats fragmentaires cassés au débitage n'ont pas été pris en compte) ; or - apparemment seule une très petite partie de ces éclats a été utilisée. A l'inverse, au moins un tiers des éclats préférentiels importés ont été manifestement utilisés et c'est dans cette catégorie que se trouvent les éclats préférentiels les plus symétriques et (globalement) les plus grands, tant dans le niveau a du Champ Bruquette que dans le niveau pléniglaciaire moyen du Tio Marché. Ainsi, le périmètre moyen des éclats préférentiels de la catégorie A (éclats abandonnés sur leur lieu même de production) est de $254 \mathrm{~mm}$, alors qu'il se monte à $275 \mathrm{~mm}$ pour les éclats de la catégorie C2 (éclats déplacés 
d'un lieu de production peut-être exogène). On peut dès lors rechercher les raisons d'un tel taux d'échec et les modalités de sa gestion ; on peut aussi tenter de dégager les principes d'un éventuel réseau de circulation entre sites, dans le cadre d'un nomadisme qui nous est largement inconnu.

La réponse à ces questions, qui touchent à l'organisation des groupes moustériens et sous-entendent des notions économiques probablement très étrangères à ces populations, ne peut provenir que de recherches menées sur l'intégralité de sites bien conservés et sur une échelle d'analyse inter-site.

Enfin, à une autre échelle, on peut se demander pourquoi certains groupes moustériens se sont tournés vers la production quasi-exclusive d'éclats Levallois préférentiels (comme à Hermies, Ault-Onival, Fitz-James, Roisel, Sainsen Amiénois, Auteuil, etc.) alors que d'autres groupes du même territoire ont fait d'autres choix tout aussi radicaux comme celui du débitage discoïde à Beauvais (Locht et Swinnen 1994). II nous semble prématuré, dans l'état actuel de la documentation, de conférer à ces choix une signification chronologique, d'autant que les gisements suffisamment bien conservés pour ne pas être qu'un bilan d'occupations diverses ne sont pas légion. A cet égard, la vallée d'Hermies montre une répétition des choix techniques, du stade $5 d$ au stade 3 , qui va à l'encontre d'un modèle chronologique trop simpliste.

\section{BIBLIOGRAPHIE}

BOEDA E. 1994 - Le concept Levallois: variabilité des méthodes. Paris : éditions du Centre National de la Recherdhe Scientifique, (Centre de Recherches Archéologiques, Monographie $n^{\circ} 9$ ).

BORDES F. 1961 - Typologie du Paléolithique inférieur et moyen. Bordeaux : Delmas, 2 vol.

CASPAR J.-P. 1988 - Contribution à la tracéologie de l'industrie lithique du néolithique ancien dans l'Europe nordoccidentale. Louvain-la-Neuve : Université Catholique de Louvain. Thèse de doctorat.

CASPAR J.-P., MASSON B. et VALLIN L. 2003 - Poli de bois ou poli de glace au Paléolithique inférieur et moyen? problèmes de convergence taphonomique et fonctionnelle. Bull. Soc. Préhist. Franç., Paris, 100 (3), p. 453-462.

CASPAR J.-P., MASSON B. et VALLIN L. 2005 - Le Moustérien à denticulés, un faciès taphonomique du Moustérien ? In : N. Molines, M.-H. Moncel et J.-L. Monnier (eds.), Les premiers peuplements en Europe. Colloque international : Données récentes sur les modalités de peuplement et sur le cadre chronostratigraphique, géologique et paléogéographique des industries du Paléolithique ancien et moyen en Europe (Rennes, 22-25 septembre 2003). Oxford : John and Erica Hedges Ltd, p. 467-478, (British Archaeological Reports, Intemational Series, S1364).
COMMONT V. 1916 - Les terrains quatemaires des tranchées du nouveau Canal du Nord. L'Anthropologie, Paris, 27 , p. $309-350$ et $517-538$.

DIBBLE H. L. et BAR-YOSEF O. (Eds.) 1995 - The Definition and Interpretation of Levallois Technology. Madison : Prehistory Press, 502 pages, (Monographs in World Archaeology, $\mathrm{n}^{\circ}$ 23).

GENESTE J.-M. 1985 - Analyse lithique d'industries moustériennes du Périgord : une approche technologique du comportement des groupes humains au Paléolithique moyen. Bordeaux : Université de Bordeaux I, 1985, 2 vol., $X+567$ pages. Thèse N.D. : Sc. : Bordeaux I ; 2 .

HEINZELIN de BRAUCOURT J. 1962 - Manuel de typologie des industries lithiques. Bruxelles : Institut Royal des Sciences Naturelles de Belgique, $74 \mathrm{p}$.

JOLICOEUR P. et MOSIMANN J.E. 1960 - Size and shape variation in the painted turtle. A principal component analysis. Growth, déc. 1960, 24, p. 339-354.

LOCHT J.-L. (éd.) 2002 - Bettencourt-Saint-Ouen (Somme). Cinq occupations paléolithiques au début de la demière glaciation. Paris, éd. Maison des Sciences de l'Homme, 172 pages, (Documents d'Archéologie Française, $n^{\circ} 90$ ).

LOCHT J.-L. et SWINNEN C. 1994 - Le débitage discoïde du gisement de Beauvais (Oise) : aspect de la chaîne opératoire au travers de quelques remontages. Paléo, 6, p. 89-104.

MASSON B. et VALLIN L. 1993 - Un atelier de débitage Levallois intact au sein des loess weichséliens du Nord de la France à Hermies (Pas-de-Calais). Bull. de la Soc. Préhist. Franç., 90 (4), p. 265-268.

MASSON B. et VALLIN L. 1996 - Ein unverlagter Schlagplatz für Levalloisabschläge im Weichselzeitlichen Löss bei Hermies (Pas-de-Calais, Frankreich). Archäologisches Korrespondenzblatt, 26, p. 225-231.

MAZIERE G. 1984 - La pièce esquillée, outil ou déchet ? Bull. de la Soc. Préhist. Franç., 81 (6), p. 182-187.

PLOUX S. 1989 - Approche technologique de la variabilité des comportements individuels : l'exemple de quelques tailleurs magdaléniens à Pincevent. Paris : Université de Paris X-Nanterre, 1989, 2 vol., 357 pages. Thèse N.D. : Lettres : Paris $\mathrm{X}$.

SALOMON A. 1913 - Découverte d'un troisième gisement moustérien sur le territoire d'Hermies au lieu-dit la vallée de Bertincourt. Bull. de la Soc. Préhist. Franç., 10, p. 191-196.

SANDGATHE D. M. 2005 - Examining the Levallois Reduction Strategy from a Design Theory Point of View. Oxford : John and Erica Hedges Ltd, 176 pages, (British Archaeological Reports, International Series, S1417). 
SEMENOV S. A. 1970 - P rehistoric Technology.2e édition. Bath: Adams \& Dart, 211 pages.

TIXIER J. 1959 - Les industries lithiques d'Aïn Fritissa (Marœ oriental). Bulletin d'Archéologie Marocaine, 3, 1958-1959, p. 107-248.

TIXIER J. 1963 - Typologie de l'Epipaléolithique du Maghreb. Paris : Arts et Métiers Graphiques, 211 pages, (Mémoires du Centre de Recherches anthropologiques préhistoriques et ethnographiques, 2).

VALLIN L. et MASSON B. 1996 - Hermies, 'Le Champ B ruquette': a site of specialized lithic production from the weichselian Middle Palaeolithic. In : F. Facchini, A. Palma di Cesnola, M. Piperno et C. Peretto (eds.), XIII U.I.S.P.P. Congress Proceedings 2, Forli, 8-14 Septembre 1996. Forli: A.B.A.C.O. Edizioni, p. 227-238.
VALLIN L. et MASSON B. 2000 - Le gisement moustérien d'Hermies "le Tio Marché " (Pas-de-Calais, France). Notae Praehistoricae, 20, Namur, p. 49-59.

VALLIN L. et MASSON B. 2004 - Behaviour towards lithic production during the Middle Palaeolithic : examples from Hermies le Champ Bruquette and Hermies le Tio Marché (Pas-de-Calais, France). In : E. A. Walker, F. Wenban-Smith et F. Healy (Eds.), Lithics in Action, Papers from the conference Lithic studies in the year 2000. Oxbow Books, p. 625, (Lithic Studies Occasional Papers, $n^{\circ} 8$ ).

VALLIN L., MASSON B. et CASPAR J.-P. 2001 Taphonomy at Hermies, France: A Mousterian Knapping Site in a Loessic Context. Joumal of Field Archaeology, 28, p. $419-436$.

\section{ANNEXE : STRUCTURE DE LA BASE DE DONNÉES}

Notre base de données sur les éclats préférentiels inclut les caractères morpho-techniques du support, les caractères morphologiques des bords et leurs caractères fonctionnels, tant macroscopiques que microscopiques. Afin de se prêter à une analyse multivariée, la table de données n'inclut que des champs de mesure et des descripteurs traités de façon binaire en terme de présence (codifiée 1)/absence (codifiée 0).

\section{Caractères du support}

\section{Caractéristiques morphométriques}

- longueur en mm,

- largeur maximale en $\mathrm{mm}$,

- épaisseur maximale en $\mathrm{mm}$,

- poids en g,

- longueur totale du pourtour en mm (mesurée au curvimètre sur dessin),

- longueur du pourtour (sans le talon) en mm (mesurée au curvimètre sur dessin),

- aire totale de l'objet en projection (en $\mathrm{cm}^{2}$ ) (calculée par un Système d'Information Géographique à partir des dessins numérisés),

- longueur radiale (en mm) depuis l'épicentre de l'objet jusqu'au pourtour, tous les $10^{\circ}$ dans le sens horaire (soit 36 mesures, calculées par un SIG à partir des dessins numérisés ; la séquence obtenue permet de codifier assez fidèlement le contour de l'éclat).

VAN PEER Ph. 1992 - The Levallois Reduction Strategy. Madison : Prehistory Press, 137 pages, (Monographs in World Archaeology, $n^{\circ} 13$ ).

\section{Caractéristiques techniques}

- extension relative du cortex sur la face dorsale, mesurée en sixièmes et codifiée en conséquence : 0/0.5/1/2/3/4/5/6,
- nombre de cicatrices d'enlèvements antérieurs,

- présence/absence (codifiée 1/0) d'accidents de débitage :

- réfléchissement

- outrepassage,

- surface de fracture pré-existante au débitage,

- ordre dans la séquence de réduction : $1^{\mathrm{er}}$ ordre ou $2^{\mathrm{e}}$ ordre (les éclats de $3^{\mathrm{e}}$ ou $4^{\mathrm{e}}$ ordre ont été catalogués de $2^{\mathrm{e}}$ ordre), - profil longitudinal : rectiligne, arqué, plongeant en partie distale ou rebroussé en partie distale (code 0/1 pour chaque possibilité).

\section{Caractéristiques taphonomiques}

- présence/absence de cassure (codifiée 1/0), que celle-ci soit survenue pendant ou après le débitage, dans la mesure où la différence ne peut pas toujours être faite,

- présence/absence (codifiée 1/0) d'altération microscopique de la surface.

Soixante-neuf objets ne présentent aucune trace d'altération, deux présentent une altération moyenne d'origine mécanique qui rend impossible la lecture de micro-traces éventuelles ; l'altération mécanique intense concerne deux pièces dont une est rendue illisible pour le tracéologue, et trente-huit pièces sont patinées (altération d'origine chimique), dont quatre sont illisibles.

\section{Caractères morphologiques du pourtour}

Afin d'apprécier les potentialités des parties actives des éclats, nous avons divisé le pourtour de chaque produit en cinq zones, numérotées dans le sens des aiguilles d'une montre et définies comme suit (fig. 25) :

Zone 3 : bord gauche proximal ; les limites sont d'une part l'extrémité gauche du talon, d'autre part une rupture dans la délinéation du bord gauche ou, à défaut de discontinuité, la moitié de la longueur de ce bord.

Zone 4 : bord gauche distal, délimité par les zones 3 et 6 . Zone 6 : bord distal, opposé au talon et délimité par une rupture dans la délinéation, de part et d'autre de l'axe du 
débitage ou, à défaut de discontinuité, défini par l'extension latérale de l'ondulation la plus éloignée du talon sur la face d'éclatement.

Zone 8 : bord droit distal, délimité par les zones 6 et 9 .

Zone 9 : bord droit proximal : les limites sont d'une part l'extrémité droite du talon, d'autre part une rupture dans la délinéation du bord droit ou, à défaut de discontinuité, la moitié de la longueur de ce bord.

Pour chacune de ces zones, nous avons relevé les paramètres suivants :

- délinéation (codifiée de façon numérique) : rectiligne, concave, convexe, sinueux (convexo-concave), festonné (succession de concavités) ou arc brisé (deux segments convexes formant entre eux un angle marqué),

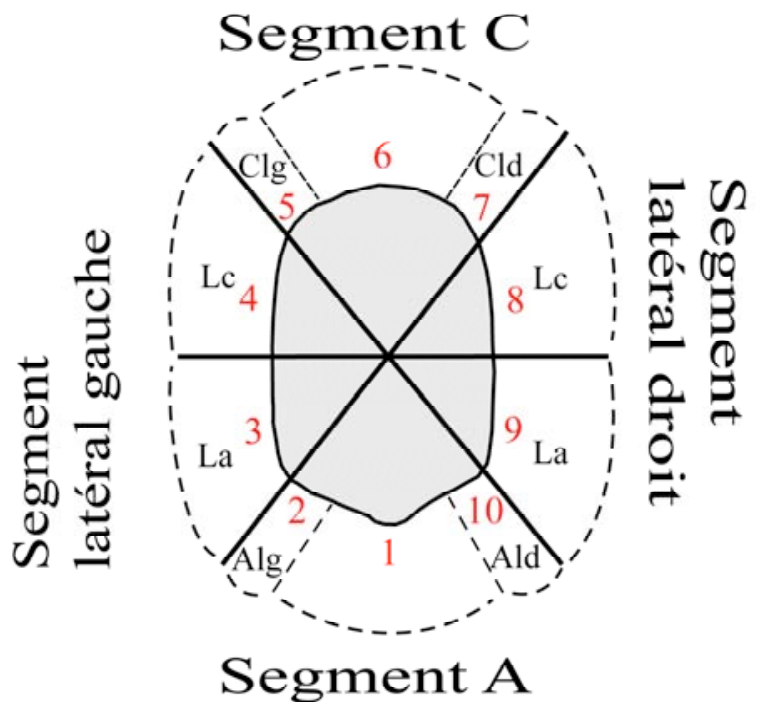

Figure 25 - Terminologie utilisée pour la description des segments de bord (d'après Boëda 1994, modifié).

Figure 25 - Used words for describing the parts of edge (from Boëda 1994, modified).
- longueur du tranchant vif (mesurée en millimètres sur le dessin, à l'aide d'un curvimètre),

- angle du tranchant vif (mesurée au rapporteur d'angle).

D'autres critères, non exclusifs entre eux et indépendamment de la délinéation de la zone de bord, ont été pris en compte en terme de présence/absence (codifiée 1/0) :

- bord modifié (que la modification soit d'origine anthropique -esquillement, retouche- ou taphonomique -piétinement, chocs au sein du sédiment),

- bord présentant un défaut (quel qu'il soit : fossile, nodule, surface de fracture, accident,...),

- bord cortical.

Par ailleurs nous avons distingué, sur le pourtour des éclats, des zones 2 et 10 qui sont respectivement les limites gauche et droite du talon, de façon à noter la présence (code 1) ou l'absence (code 0) de fines retouches régulières en ces points.

\section{Caractères fonctionnels des bords}

Chaque éclat préférentiel a été soumis à un balayage systématique des bords au microscope métallographique par l'un d'entre nous (J.-P. C.). Pour chaque zone de bord, un critère " bord utilisé " a été renseigné (en terme de présence/absence). Un certain nombre de réserves doivent cependant être émises quant à l'interprétation des résultats de l'examen microscopique ; certaines pièces ont pu être utlisées sans enregistrer de traces perceptibles, soit parce que la surface est altérée (cela concerne 7 individus, cf. supra), soit parce que l'utilisation a été trop brève ou trop faible, soit parce que le bord utilisé présente une texture saccharoïde inapte à l'enregistrement des actions.

A partir de l'examen microscopique et de l'examen macroscopique, deux critères fonctionnels (non exclusifs entre eux) ont été renseignés pour chaque individu en terme de présence/absence :

- macrotraces (retouches irrégulières, assez envahissantes, écailleuses ou scalariformes, bifaciales ou alternantes),

- microtraces (micropoli d'usage, quel qu'il soit). 